\title{
Some insights into computational models of (patho)physiological brain activity
}

\author{
Piotr Suffczynski, Fabrice Wendling , Jean-Jacques Bellanger, Fernando H. Lopes da Silva
}

P.S is with Laboratory of Biomedical Physics, Institute of Experimental Physics, Warsaw University, Hoza 69, 00-681 Warsaw, Poland. Phone: + 4822 6254535, fax: + 4822 5532320, email: suffa@fuw.edu.pl

F.W and J-J. B are with INSERM U642 - Universite de Rennes 1, Laboratoire Traitement du Signal et de L'Image, Campus de Beaulieu - Batiment 22, 35042 Rennes Cedex, France.

F. LdS is with Centre of NeuroSciences, Swammerdam Institute for Life Sciences, University of Amsterdam, Kruislaan 320, 1098 SM, Amsterdam, The Netherlands.

\begin{abstract}
The amount of experimental data concerning physiology and anatomy of the nervous system is growing very fast, challenging our capacity to make comprehensive syntheses of the plethora of data available. Computer models of neuronal networks provide useful tools to construct such syntheses. They can be used to interpret experimental data, generate experimentally testable predictions and formulate new hypotheses regarding the function of the neural systems. Models can also act as a bridge between different levels of neuronal organization. The ultimate aim of computational neuroscience is to provide a link between behavior and underlying neural mechanisms. Depending on the specific aim of the model, there are different levels of neuronal organization at which the model can be set. Models are constructed at the microscopic (molecular and cellular), macroscopic level (local populations or systems) or dynamical systems level. Apart from purely computational models, hybrid networks are being developed in which biological neurons are connected in vitro to computer simulated neurons. Also, neuromorphic systems are recently being created using silicon chips that mimic computational operations in the brain. This article reviews various computational models of the brain and insights obtained through their simulations.
\end{abstract}

Index Terms- Brain modeling, computational neuroscience, nervous system, simulation

\section{INTRODUCTION}

Computational neuroscience is an interdisciplinary field connected to neuroscience, biophysics, applied mathematics and computer science. In essence it uses mathematical and computational techniques such as computer simulation to understand the function of the nervous system.

The central nervous system - the brain - is considered to be the most complex system in nature. Therefore understanding its functions offers a great challenge to biological and physical sciences. Neurobiological research yields new and detailed knowledge at a very high rate but it is becoming clear that to understand how the brain works it is not enough to accumulate continuously more and more facts. In order to acquire a coherent view of the mechanisms through which the nervous system mediates behavior, the experimental facts have to be related to each other. The interactions between neural components that underlie a given behavior can be studied at various levels of neuronal organizations, from genes and molecules to whole systems that mediate specific functions such as vision or balance. The multilevel hierarchy of the brain and how, in general terms, the communication between nerve cells takes place, is depicted in Fig. 1. Since behavior depends on the coordinated action of neural elements both within and across different levels, understanding how the brain mediates behavior requires across-scale integration of the knowledge obtained at different levels of neuronal organization. It is being recognized that due to the complexity of neural interactions a formalized approach is necessary in order to develop a scientific theory that relates experimentally observable facts within a consistent framework. In many instances, models of neural mechanisms based on computer simulations provide an important step in acquiring such a synthetic view. In general, a theory can be either phenomenological, accounting for the relationships between experimentally observed variables or mechanistic, aiming at explaining a given phenomenon in terms of realistic mechanisms. In case of simulations of brain functions, mechanistic (or realistic) models often involve also phenomenological descriptions, at least partly, and therefore the straightforward division of neural models into two distinct classes is not always possible ([2]).

Computational brain models may be used to reproduce a particular observed behavior. This capacity of a model is what we may call its face value. However this should not be the sole purpose of a model. It is equally important, or even more important, that a model explicitly incorporates features that can reproduce natural processes likely to play role in the real system. This is what we may call the constructive value of the model. Therefore, the validation of the model cannot be achieved in isolation to real experiments. Furthermore, the

Fig. 1 over here

model should be able to generate predictions about features of the real system that were not originally recognized. This is 
the predictive value of the model. These novel features, in principle, can be tested experimentally. The confirmation of the model's predictions increases confidence in its value, while their refutation implies that a revision of the model and/or new experiments is necessary. Indeed such a validation procedure can motivate new experiments that have to be performed to discern between competing hypotheses. The acquired experimental knowledge can be used in turn to develop the model further. Therefore, the two way interaction between modeler and experimenter is essential for the advancement of neuroscientific research.

A model is always a simplification of the real system. Modeling is essentially a reductionistic approach, where the question "How far can we simplify?" occupies a central place. There are different approaches to answer this question. Some authors believe that only models with a high degree of realism are likely to give us insight into neural mechanisms. Other authors, on the contrary, follow a parsimonious approach which minimizes model's complexity. This is usually achieved by starting with a minimal set of features of the modeled system and adding subsequent features as necessary to obtain the desired model behavior. In selecting the degree of biological realism and the level of neuronal organization at which the model is constructed, there are also other factors that should be taken into account. Among these are: the level of detail required to account for a particular phenomenon, the nature of available real observations necessary to choose reliable parameter values and to validate modeling results, the computational efficiency with respect to available computing power. In general, modeling may also be used as a way to integrate different levels of description. To achieve such synthesis, models must be organized according to different levels of detail and complexity, from the microscopic to the macroscopic levels.

The intent of this paper is to provide a brief review of the most relevant modeling studies that explored neural computations in several brain systems at different scales. We provide also some specific examples of how models are created, explored, evaluated and ultimately used to generate novel hypotheses. In this context special emphasis is placed on neural population models that were recently developed to progress our understanding of mechanisms involved in the generation of epileptic activity.

\section{BRIEF HISTORICAL BACKGROUND}

\section{A. McCulloch and Pits' neuron}

McCulloch and Pits's approach ([3]) was inspired by Kant and Leibnitz, seeking to map logic onto neural function. Hence, the corresponding phenomenological model is a very simple and highly formalized model which is based rather on a coarse structure of biological neurons and on desired logical functionality, than on physiological properties. A McCullochPits neuron has multiple inputs and a single output. Each input is weighted, and the weighted sum of these inputs is passed through a nonlinearity to obtain the output of the model. In fact this particular model is a neural model with synaptic inputs (inhibitory when the weight is negative, excitatory when the weight is positive), simplified somatic threshold and saturation effects, and axonal output 'activity' resumed as a static function of input activities. Although McCulloch-Pits model can solve pattern classification problems (and thus emulate an important feature of natural intelligence), it is no longer considered a useful model for interpreting real neurophysiological data.

\section{B. Rall's theory}

Rall's theory describes the propagation of electrical stimuli from the site of dendritic synaptic inputs along passive dendritic trees to the soma. This theory is based on "cable theory" developed by W. Thomson (1824-1907), later Lord Kelvin, to model spatio-temporal evolution of voltages and currents in the first transatlantic cable around 1855. In analogy with a cylindrical conductor surrounded by a leaky insulator and immerged in a conductive environment, a piece of dendritic membrane is viewed as a 'leaky' pipe through which transversal ionic currents are imposed by potential differences between intra- and extracellullar space. Inside the membrane, longitudinal currents are established in the conductive active solution when the cytoplasmic potential varies along the longitudinal axis. Neglecting the transversal dimensions, a partial differential equation reduced to one spatial dimension can be established. Introduced by Hodgkin and Rushton [4] in the study of nerve fibers, cable theory was applied to neurons in the middle of the 50s ([5], [6]) to interpret experimental data obtained from individual neurons with intracellular microelectrodes located in the soma. A series of important papers by Rall ([7]-[9]) then followed. They included transient cable properties that were neglected in the first models and led to the correction of discrepancies between model and experimental results ([10], [11]).

In absence of voltage dependant currents and input (synaptic) currents, the cable equation can be can be written as

$$
\frac{\partial^{2} V}{\partial x^{2}}=r_{i}\left[I_{\text {leak }}+I_{\text {cap }}\right]=r_{i}\left[\frac{\left(V-E_{\text {leak }}\right)}{r_{m}}+C_{m} \frac{\partial V}{\partial t}\right](1)
$$

This equation is obtained from a discrete space decomposition of the cable in elementary units (see caption of Fig. 2 for definition of quantities). It can be analytically solved for specific boundary conditions and injected current step at $x=0$. Assuming equipotential cytoplasmic medium, $V(t)$ is given by $V(t)=V_{0} \exp \left(-t /\left(r_{m} \cdot C_{m}\right)\right)$ and, in the steady state limit, $V(x)=A_{1} \exp (x / \lambda)+A_{2} \exp (-x / \lambda)$ where $\lambda=\sqrt{r_{m} / r_{i}}=\sqrt{d \cdot R_{m} / 4 . R_{i}}$ and where $\mathrm{A}_{1}, \mathrm{~A}_{2}$ and $\mathrm{V}_{0}$ depend on injected current level and boundary conditions.

Moreover, a dendritic network has the spatial structure of a Fig. 2 over here

tree. Although each model branch can be simplified to a homogeneous piece of cable with given global characteristics 
(geometrical and electrical), the analytic calculation of $V(x, t)$ in time and elsewhere in the tree can be quite difficult. Conditions have been introduced by Rall ([8]) that considerably simplify the calculation of $V(0, t), \quad x=0$ corresponding to the soma (the root of the tree): each branch of the dendritic network tree is considered as a set of equivalent cylinders, the complete dendritic network is then equivalent to a single cylinder. The soma is assumed to be isopotential and to behave like a resistance and a capacitance in parallel and is connected to one of the two extremities of the single cylinder. The other extremity corresponds to a lumped input.

\section{Hodgkin - Huxley theory}

In the early 50's, Hodgkin and Huxley developed a model (referred to as the $\mathrm{HH}$ model in the following) to explain observed patterns of action potentials in the giant axon of the squid. As for Rall's model, the HH model considers a small portion of membrane and its equivalent electrical circuit. However, in the basic $\mathrm{HH}$ model, a null longitudinal cytoplasmic current is assumed (equipotential cytoplasm). Experimental conditions satisfying this hypothesis are realised by mean of the space clamp technique to maintain a uniform spatial distribution of membrane potential $(V)$ over an extended region of the cell where currents are recorded. Moreover, in addition to the pair $\left(E_{\text {leak }}, r_{m}\right)$ which represents the total trans-membrane ionic current in the passive cable model, two other pairs $\left(E_{K}, r_{K}=1 / g_{K}\right)$ and $\left(E_{N_{a}}, r_{N_{a}}=1 / g_{N_{a}}\right)$ are introduced to model potassium and sodium specific ionic currents, respectively (Fig. 1b). With the new ionic currents, voltage $V$ is described by the equation:

$$
C_{m} \frac{d V}{d t}=\sum_{x=l e a k, K, N_{a}} g_{x}(V(t), t)\left(V(t)-V_{x}\right)+I_{i n j}(t)
$$

where $I_{i n j}$ denotes an injected current (synaptic current or experimental stimulation). Historically, Hodgkin and Katz ([12]) first demonstrated that both sodium and potassium make significant contributions to the ionic current underlying the action potential. Following these works, Hodgkin and Huxley found that the permeability of the membrane to specific ions was a function of both time and $V$ and presented their results in their famous paper published in 1952 ([13]). The heart of the HH model is a description of the time- and voltage-dependent conductances for sodium and potassium as a function of gating variables $m, h$, and $n$ : $g_{N a}=\bar{g}_{N a} \cdot m^{3} h, g_{K}=\bar{g}_{K} \cdot n^{4}$. These variables can be interpreted as a fraction of gates being in permissive state (hence, they can vary from 0 to 1 ), whereas $\bar{g}_{N_{a}}$ and $\bar{g}_{K}$ represent maximal values. They can be of activation type (conductance increases with depolarization) or inactivation type (the converse). They obey kinetic equations ( $p_{i}$ is for $m$, $h$, or $n) \frac{d p_{i}}{d t}=\alpha_{i}(V)\left(1-p_{i}\right)-\beta_{i}(V) p_{i}$ equivalent to $\tau_{i}(V) \frac{d p_{i}}{d t}+p_{i}=\alpha_{i}(V) /\left(\left(\alpha_{i}(V)+\beta_{i}(V)\right) \quad\right.$ with $\tau_{i}=1 /\left(\alpha_{i}(V)+\beta_{i}(V)\right)$ (bell shape curve-fitting). These equations correspond to first order differential equations with voltage dependant time constants $\tau_{i}(V)=1 /\left(\alpha_{i}(V)+\beta_{i}(V)\right)$ and voltage dependant steady state values (when they exist) $p_{i S}(V)=\alpha_{i}(V) /\left(\left(\alpha_{i}(V)+\beta_{i}(V)\right) \quad\right.$ (sigmoid shape curve-fitting). As demonstrated by computer simulations, the $\mathrm{HH}$ model generates action potentials of appropriate shape, threshold and refractory periods (both absolute and relative). Further developments also added other active ion channels or temperature effects to the model. Some simplified versions of HH model were also proposed. FitzHugh ([14]) and Nagumo ([15]) showed that, for a purpose of action potential generation, $\mathrm{HH}$ model of order four can be reduced to a model of order two which highly simplifies its dynamical study. Interestingly, this phenomenological model and other simplified neuronal models (Morris-Lecar ([16]), integrateand-fire (a survey can be found in [17])) gain interest over the past decade due to their simplicity and efficiency in studying dynamical properties of single neurons, simplified neuron interactions in large networks and brain information coding or decoding (section IV.C). Finally, it is important to underline that advanced single channel recording techniques were developed decades after the HH model and that they permitted some model assumptions (regarding the nature of microscopic ionic channels) to be verified. Recent biophysical models of ionic channels are presented in the next section.

\section{MOLECULAR AND CELLULAR MODELS}

\section{A. Ionic channels, membranes, synapses}

Ionic channels play a crucial role in the physiology of complex biological systems such as the brain. Therefore theories and computational models are being developed to clarify the function of ion channel processes such as permeation, selectivity and gating. A microscopic model of ionic permeation in the selectivity filter of ionic channels has been developed by $\mathrm{Wu}$ ([18]). The model is based on molecular kinetic theory and makes many specific assumptions about ion - environment interactions (e. g., ions move independently, ion movement is near the axis of the channel, etc.). The model predicts that channel radius and ionwater interactions are two major channel structural determinants of ion selectivity. The studies aimed at understanding the relationship between structure and function of membrane proteins is currently being carried out using computational tools by Sansom and co-workers ([19], [20). Research of this group focuses on potassium channels, both voltage gated and ligand/protein gated, nicotinic acetylcholine 
receptors and glutamate receptors, with special emphasis on gating mechanisms. This computational approach allows to bridge several time scales, from sub-nanoseconds protein motions to milliseconds time scale motions involved in channel gating. Understanding the functions of membrane proteins is also important from a biomedical perspective, since ionic channels and receptors are possible targets for novel drugs acting on the nervous systems ([21]). A mathematical analysis and numerical simulations of the channel dynamics is presented in [22]. This shows that channel activity can be approximated by a one-dimensional bistable Langevin equation. These analytical results, which were in good agreement with numerical simulations, suggested that spontaneous action potentials generation can arise from channel fluctuations. Also the factors determining the threshold for action potential initiation in neurons were investigated using a computational model ([23]). The simulations didn't confirm the traditional view that a high density of sodium channels in the initial segment of the axon determines the lowest threshold. Instead they pointed to biophysical properties of axonal channels that are critical to action potential initiation. Initiation of dendritic spikes was investigated in a computational study by Mainen ([24]). Apart from models of membranes with voltage dependent conductances, the passive membrane properties have also been modelled. The spike initiation and propagation was investigated in the model of axon ([25]). The axon had a slow regenerative conductance as well as the usual $\mathrm{HH}$ type sodium and potassium conductances (section II.C). This study showed how complex dynamics can arise in excitable membranes with fast and slow conductances. Passive neuronal models were also used to study how synaptic conductance waveforms and intrinsic membrane properties affect the excitatory postsynaptic potential (EPSP) and action potential generation ([26]). This study showed that developmental changes in EPSP shape increase the temporal precision of spike generation thus contributing to fidelity of information coding in the brain. Computational models of axons were used also to investigate nerve stimulation under magnetic resonance imaging (MRI) magnetic fields ([27]). Axons were represented by a one-dimensional compartmental cable model including the kinetics of mammalian myelinated fibres. By solving the cable equation along the axons in the presence of magnetic field gradients the authors were able to test several axon and field parameters in order to determine the threshold values for axonal stimulation in patients undergoing a MRI scan.

As far as synapses are concerned, a model of transmitter release is provided in [28] while a comprehensive general description of synaptic transmission with Markov kinetic models and practical algorithms is presented in [29]. Models that made use of this formalism are e.g., [30] in which the authors demonstrated that randomly fluctuating inputs from glutamatergic (i.e, using glutmate as neurotransmitter) and GABAergic (i.e., using gamma-aminobutyric acid as neurotransmitter) neurons can account for several properties (e.g., irregular spontaneous firing activity) of cortical neurons observed in vivo. However, these models do not deal with the stochasticity of individual synapses although synaptic transmission is an inherently stochastic process. The theoretical formulation of dynamic stochastic synapses has been carried out in [30].

Finally, changes of the amplitude of postsynaptic response to an incoming action potential of constant amplitude can be manifestations of synaptic plasticity. A persistent increase of synaptic transmission efficacy is called long-term potentiation of synapses, or LTP for short, while a persistent decrease of synaptic efficacy, is called long-term depression (LTD). A phenomenological model of both long-term potentiation and long-term depression is presented in [32]. In that model the change of synaptic strength is introduced using two variables: the first one dependent on presynaptic voltages, the second one dependent on postsynaptic actions. The resultant LTP or LTD from the joint action of these two processes depends on the relative timing between them. The dynamical model reproduces the spike time-dependent plasticity of excitatory synapses as a function of relative timing between pre- and postsynaptic events. Apart from accounting for experimental observations the model gives also predictions for changes in synaptic strength when periodic spike trains of varying frequency, and Poisson distributed spike trains with varying average frequency are presented pre- and postsynaptically. An analytical study and VLSI (Very Large Scale Integrated) implementation of the plastic synapse is presented in [33]. More detailed, biophysical models of synaptic plasticity have also been developed ([34] - [38]). These models contain two essential components, a description of intracellular calcium dynamics, in particular a model of calcium entry through $\mathrm{N}$ methyl D-aspartate (NMDA) synapses, and a hypothesis of how the concentration of intracellular calcium influences the change of synaptic efficacy. Modeling studies that included changes of synaptic efficiencies are reviewed in [39], [40]).

\section{B. Models with glia and extracellular space}

It has been known for many years that glia, the most abundant cell type in the brain, play more than a kind of 'glue' role for the surrounding neurons. Similarly, it is widely accepted that the composition of extracellular space (e.g., external calcium concentration) sets the conditions for neural activity and neurotransmission. However, most computational studies take only the neurons and their connections, under stationary external conditions, in consideration, and only a minority of models considers the role of glia and extracellular space in functioning of the brain.

The computational work of Wiest ([41]) has been motivated by experimental observations that normal neural activity is associated with local changes in extracellular calcium concentration. Using realistic calcium channel models, experimentally measured back-propagating action potentials, and a model of the extracellular space, the fluctuations in external calcium during neural activity were computed. A significant extracellular calcium drop was shown to result 
from coincident spikes arriving at dendrites that shared the same tissue volume. These results pointed to an alternative way of how information processing may take place in the brain. Another computational study of the same group ([42]) showed that decrements in external calcium levels may mediate a form of short-term synaptic depression.

The effect of spreading depression in relation to epileptiform neuronal activity was investigated in models by Kager ([43], [44]). A realistic model of hippocampal pyramidal cell included an extracellular compartment and a buffering process provided by glial cells. The simulations showed that the spreading depression phenomenon is dependent on glial reuptake of extracellular potassium and can be generated by the feedback of ionic currents that change extracellular ion concentrations, which, in turn, influence ionic currents and membrane potentials. These results pointed to the essential role of glial cells in the maintenance of normal activity of the brain. Other studies confirmed a pivotal role of glial cells in preventing spreading depression and additionally pointed to potassium movement through gap junctions as a necessary component of this process ([45]).

A computational model of extracellular local field potentials has been elaborated by Bedard ([46]). In that model the ion concentrations in the extracellular space were not homogeneous, what led to the attenuation of the local field potentials with distance from the source, in a frequency dependent manner. The low pass filtering properties of the extracellular space predicted by the model were in good agreement with experimental observations.

\section{NEURAL NETWORKS MODELS}

\section{A. Single cell models}

Single cell models use compartments to represent some characteristics of the 3D anatomy and the function of the neuron. Model parameters are related to morphology, passive (membrane capacitance, axial resistivity, leak conductance, membrane time constant, leak reversal potential) and active electrical properties (i.e. ion channels that are specific to the cell type and that obey current balance equations, see section II.C). In more detailed versions (that may contain several thousands compartments), some refinements were proposed in the representation of the initial segment and axon, the latter having been shown, in some cases, not to stem from the soma body but rather from a dendrite slightly away from the soma ([47]).

Neuron models provide a way to explore some parameters that still remain difficult to identify based on experimental observations, such as the relative densities of specific ionic channels in particular regions of a neuron or to investigate the role of voltage-dependent ion channels in shaping cellular activity. The first models were developed by Rall and Shepherd ([48]) to represent passive electrical properties. Then, in order to add active properties and to test some hypotheses about differences in impulse responses at the soma and at the axon, Dodge and colleagues ([49], [50]) proposed a computer simulation of a motoneuron in which thresholds in these regions could be altered by using different sets of values for the voltage sensitive rate constants (in the $\mathrm{HH}$ equations) for the soma and axon.

Whole cell models were proposed by Traub and colleagues for neocortical and hippocampal pyramidal cells ([51] - [53]). In such models, the ionic channels in each compartment are represented by differential equations describing their properties (whether voltage-gated, ligand-gated, or second messenger-activated properties). These early simulations identified processes at the membrane level that may be responsible for the generation of intrinsic epileptiform bursts in single cells. It allowed to perform subsequent simulations of abnormal synchronization of large networks that are reviewed in the next section (IV.B). In a more recent study of cortical cells ([54]) the authors have shown that changes in the level of membrane potential may make the cell switch between different firing patterns (rhythmic spiking, rhythmic bursting and fast spiking). This study, confirmed by experimental observations, pointed against the traditional division of neocortical cells into fixed categories based on their firing properties.

A number of computational studies were carried out to elucidate the influence of various ionic conductances on the activity of thalamic neurons. These neurons exhibit a variety of activity modes depending on membrane potential level and external stimulation pattern. The currents implicated in the activity of thalamic relay neurons were simulated in [55] while rhythmic firing patterns of these cells were simulated in an accompanying study ([56]). Wang ([57]) thoroughly studied a thalamic relay neuron model and showed that this cell may generate a whole spectrum of activities from intrinsic oscillations at various frequencies to chaotic behavior. Properties of thalamic reticular cells were investigated e.g. in a compartmental model by Destexhe [58]. In order to reproduce experimental observations, it was necessary to include a high density of dendritic calcium currents in the model neuron. This study provides an excellent example of how a computational model may be used to predict the spatial distribution of specific ionic channels densities. A single cell model recently developed by the Destexhe's group ([59]) focused on the effect of background synaptic noise on input/output properties of neocortical cells.

Single cell models have also been developed to study morphological and functional properties of interneurons. In the hippocampus, for instance, models including about 200 compartments were used to investigate the diversity of interneuronal cells and to relate differences of axonal and dendritic arbors, electrophysiological responses, ion channel distribution and kinetics to distinct functional roles ([60]).

A recent review was also presented by Segev and London ([61]) about the use of quantitative single cell models for studying the function of dendrites, specifically. In this paper, the authors recall some key biophysical insights gained from reduced models. More generally, they emphasize that the 
construction of an intense interaction between experimental work and modeling has now became a necessity to understand the complex dendritic machinery. The importance of this interaction was also pointed out in the computational study of De Schutter ([62]), in which a realistic model of a Purkinje cell was constructed to replicate results obtained in vitro. This model made a number of testable predictions that were subsequently tested experimentally, providing insight into synaptic integration and leading to the development of a new theory about the function of long-term depression in the cerebellum.

Another modeling study with direct experimental application was carried out in [63]. Using a modeled neuron the authors evaluated the accuracy of the voltage clamp technique used to determine the properties of voltage-gated conductances. Parameters of current - voltage curves obtained by fitting the $\mathrm{HH}$ equations to the clamp currents were compared with the values originally set in the model. Good fits were obtained for moderate channel densities while the parameter errors increased with conductance density. Significant errors arose in poorly space-clamped cells. Using another set of simulations the authors calculated the corrections for parameters of ionic currents measured with not sufficient spatial control ([64]).

\section{B. Distributed neuronal networks}

For studies of network phenomena such as those implied in epileptic discharges, models have been proposed based on the interconnections of neuron models via synaptic processes. The key idea is to describe sub-threshold potential fluctuations and firing properties of networks of neurons based on an accurate description of membrane processes (ionic currents) of the different parts of each cell, and on physiologically relevant strategies to account for the pattern of interconnections of a large number of cells. The first attempts in this context were presented by Traub and collaborators ([65]-[67]) who simulated the activity of small parts of the CA3 (CA: Cornu Amonis) of the hippocampus using networks of interconnected neurons (about 10000 cells divided into 9,000 excitatory neurons and 900 inhibitory cells), each one represented by a reduced number of compartments (equal to 19). With individual cells and synapses having properties based on experimental data, these authors studied the influence of various parameters related to synaptic strength and connectivity. In particular, they studied the relationship between spike propagation velocity throughout the neuronal array and the spatial extent of excitatory connectivity. They also determined conditions relevant for the genesis of physiological and pathological (epileptic) rhythmic activities. Investigations by Pinsky and Rinzel ([68]) then followed. Based on Traub's model, these authors proposed a reduced representation (two compartments, eight variables) of a CA3 pyramidal cell and studied its dynamics at both cellular and network level. One interesting result is that they reproduced some results obtained by Traub in [66] with a model of reduced complexity (in terms of number of compartments per cell). The next series of modeling studies carried out by Traub and coworkers ([69], [70]) addressed the question of the generation and long-range synchronization of gamma rhythms $(\sim 40 \mathrm{~Hz})$ in a neuronal network. These modeling studies showed that inhibitory interneuronal networks interacting with excitatory pyramidal cells could account for this phenomenon. Propagation of activity in purely inhibitory neural networks was investigated in [71]. Recent Traub's models ([72], [73]) concerned the role of gap junctions in the generation of very fast oscillations $(>70 \mathrm{~Hz})$ and seizure initiation. Seizure generation in hippocampal networks is also addressed in several computational models ([74], [75]).

A variety of purely cortical models have been developed recently. These models provided insight into cortically generated activity such as the slow rhythm ([76]), the alpha rhythm ([77], [78]) or processes related to vision ([79], [80]), olfaction ([81] - []83), attention ([84], [85]), memory ([86] [91]), and higher brain functions ([92], [93]).

A number of network models have also been developed to study various functional features (sleep, attention, epilepsy) mediated by thalamocortical circuitry. Early models considered a single pair of coupled thalamic relay cell and reticular thalamic cell ([94]), isolated thalamic reticular nucleus cells ([95] - [98]) or a whole thalamic network ([99] [103]). These models provided insight into thalamic pacemaker mechanisms of the generation of sleep spindles and of abnormal $3 \mathrm{~Hz}$ oscillations. Using full thalamocortical models, the synchronization of sleep spindles mediated by cortical feedback was investigated further ([104]), as well as the transition between spindles and delta rhythm ([105]) and thalamic gating by the cortex ([106]). Computer simulations of thalamocortical networks were also used to investigate network mechanisms responsible for the generation of activity associated with pathological behavior such as epileptic seizures ([106] - [110]).

Recently, Hill and Tononi ([111]) developed a large-scale model of the thalamocortical system with the objective of accounting for the transition from wakefulness to sleep and the generation of the slow oscillation at several different levelsfrom ion channel kinetics to global EEG phenomena. The model incorporates detailed features of the neuroanatomical organization of the thalamocortical system, and a large number of neurons $(>65,000)$ interconnected by millions of intra- and interareal connections. An essential aspect of the model is that at the cellular level it incorporates several types of intrinsic conductances (mediating the hyperpolarization-activated cation current $I_{\mathrm{h}}$, low-threshold calcium current $I_{\mathrm{T}}$, persistent sodium current $I_{\mathrm{Na}(\mathrm{p})}$, potassium leak current $I_{\mathrm{KL}}$, depolarization-dependent potassium current $I_{\mathrm{DK}}$-representing $\mathrm{Ca}^{2+}$ and $\mathrm{Na}^{+}$-dependent $\mathrm{K}^{+}$currents) and synaptic currents [ $\propto-$ amino-3-hydroxy-5-methyl-4-isoxazolepropionic acid (AMPA), $N$-methyl-D-aspartate (NMDA), $\gamma$-aminobutyric acid-A $\left(\mathrm{GABA}_{\mathrm{A}}\right), \gamma$-aminobutyric acid-B $\left.\left(\mathrm{GABA}_{\mathrm{B}}\right)\right]$. The model is capable of generating signals that are compatible with experimental data ranging from intracellular traces to field potentials as recoded in the EEG. Most interesting is that 
the model can switch from a waking to a sleep mode of activity, the latter being characterised by spontaneous patterns typical of wakefulness including low-voltage fast activity in the EEG, and the latter by slow oscillations that closely resemble those observed experimentally during sleep. The slow oscillation displays a bimodal membrane potential distribution, i.e. a disfacilitated and silent down-state and a depolarized, high-conductance up-state that exhibits gamma frequency synchronization. The synchronization of the slow oscillation in the model depends on corticocortical connections, consistent with experimental observations.

In this model the transitions from the awake state to slowwave sleep primarily arise from an increase in the potassium leak conductance $\mathrm{g}_{\mathrm{KL}}$, which is present in all model neurons. This may be induced by the reduced actions during sleep of neuromodulators such as acetylcholine. In short, the same model is capable, by changing a few parameters that simulate the effects of the reduced release of neuromodulators (mainly acetylcholine) on certain potassium currents, to display a transition between a waking and a sleep mode of activity. The influence of other ioinic currents was also studied in detail. Nevertheless a dynamical analysis of the model's behavior, in mathematical terms is not yet available.

\section{Spike trains}

Both spike rate and spike timing of action potentials are important determinants of information coding in the brain. However, it is still not clear what are the exact rules underlying neural coding. A large number of studies addressed questions related to elucidating this fundamental issue in neuroscience using theoretical analyses, artificial neural networks and biologically realistic models. Comprehensive treatments of research on information coding in the brain are provided e.g., in [112] - [114].

\section{NEURONAL POPULATION MODELS}

Many brain functions such as sensory information processing or pattern recognition result from large-scale spatiotemporal activity in masses of neurons in which the number of cells is so high that any approach starting at the single cell level would rapidly become intractable. This consideration was the starting point of another modeling approach which developed since the 1960's and which conceptually differed from approaches detailed in the previous sections in the sense that they emphasize the properties of populations of cells instead of those of individual neurons. In considered populations, cells are assumed to be spatially close and their interconnections are assumed to be random but dense enough so that the probability for any two cells in the population to be connected (either directly or via interneurons) is high. Based on these assumptions, population models represent the temporal dynamics of the aggregates while the spatial interactions between cells are neglected. This implies that the relevant variable of these models is not the single spike but rather the spike frequency, or firing-rate, computed from the total current delivered by synaptic inputs (which sum linearly according to the mean-field approximation) into the soma. Neuronal population models have been advanced in numerous forms during the past decades by physiologists, psychologists and theorists. In the following, we only refer to pioneer works (performed in the early 1970's) of Wilson and Cowan (section V.A) who put the theoretical bases of these models and works of Freeman et al. (section V.B) and Lopes da Silva et al. (section V.C) who first applied this modeling approach to answer specific questions raised by neurophysiological observations.

\section{A. Wilson and Cowan's model}

In the early 70's, Wilson and Cowan ([115) proposed a set of nonlinear differential equations to account for the dynamics of a localized population of neurons. Briefly, they started from physiological evidence regarding the existence of such populations and from a crucial assumption, considered, at that time, as an axiom: all neural processes depend upon the interaction of excitatory and inhibitory cells. Therefore, they considered a population as being composed of two subpopulations, one excitatory and the other one inhibitory and proposed an approach based on two variables, representing the proportion of excitatory and inhibitory cells firing per unit time, respectively. This two-variable approach was fundamentally different from those used in other studies of neural populations until then. Using a sigmoid shape for each subpopulation response, they were able to derive general equations governing the temporal dynamics of a population of neurons containing excitatory and inhibitory subpopulations. Using phase-plane analysis, they also studied simple and multiple hysteresis phenomena and limit cycle activities represented in the model and they managed to relate their results to a number of experimental findings like those of Freeman ([116]) that were obtained using electrophysiological recordings in the prepyriform cortex of cats.

\section{B. Olfactory system - Freeman's model}

Since the early 60's, Freeman and colleagues have developed a comprehensive model describing the global organization of the olfactory system ([116] - [118]). Besides the need to better understand the olfaction function, one of their goals was also to relate some physiological data recorded in animals to mechanisms involved in sensory or perception processing. Briefly, the model input is represented by the activity of receptors located in the nasal mucosa and connected to the primary olfactory nerve which terminates on the anterior olfactory nucleus (AON) and on the olfactory bulb (OB). The output of the bulb is fed into the prepyriform cortex (PC). Pyramidal cells of the PC are the output neurons for more distant cortical areas (entorhinal cortex). Each part (AON, OB and PC) contains four subsets of cells (two excitatory and two inhibitory). The first key feature of Freeman's model is that these subsets are "lumped" representations of aggregates of strongly interconnected neurons (i.e. neural masses) which have, on average, similar properties such as a common sign of output (i.e. they are 
either all excitatory or all inhibitory). The second key feature is that the dynamics of each subset are simply described by a second order nonlinear ordinary differential equation (ODE) representing conversion operations between the population average postsynaptic potential ("wave") and the density of action potentials ("pulse") fired by local neurons. This ODE comes from the linear transfer function (of order 2) used to transform the average pulse density of afferent action potentials into an average post-synaptic membrane potential ("pulse-wave") and from a static (i.e. not time-varying) nonlinear function used to model the relationship between the average post-synaptic potential of a given subset and the average pulse density of potentials fired by the neurons ("wave-pulse"). Linearity of the pulse-wave function is related to the spatial and temporal integration performed by the dendrites (superposition). Nonlinearity of the wave-pulse function (asymmetric sigmoid curve, [119]) was demonstrated experimentally based on simultaneous measurements of pulse and wave activity from the same population.

The authors also justify that it is a static relationship in view of two arguments: i) individual neurons in the population fire unpredictably (Poisson distribution of occurrence times) and ii) they also fire in an uncorrelated manner with respect to each other. Through this macroscopic level of modeling, Freeman and colleagues managed to establish some relationships between EEG dynamics and behavioral functions (such as sensory reception and perception) related to state variables of the model. Indeed, assuming time constants fixed at physiologically determined values in the pulse-wave conversion, gain constants fixed at a common value in the wave-pulse sigmoidal relationship and appropriate setting of connection strengths between nodes within the $\mathrm{AON}, \mathrm{OB}$, and PC, they showed that the model produces realistic EEG signals that approximate those experimentally recorded in these brain structures ([120]).

\section{Models of rhythmic activity in the thalamus and cortex -} Lopes da Silva's models

The alpha rhythm model was originally developed as a possible explanation of the origin of the alpha rhythm in dog by Lopes da Silva and collaborators about 30 years ago. This model, as described in the original publication ([121]), consisted of two parts. The first part described the distributed network model where each neuron was modeled individually. Subsequently the distributed model was converted to the lumped populations form what allowed to follow a system analysis approach to analytically evaluate the influence of different neurophysiological parameters upon the statistical properties of the output rhythmic activity.

The population alpha rhythm model was based on two interacting populations of neurons. They represented the populations of excitatory thalamocortical relay cells (TCR) and of inhibitory interneurons (IN) that were interconnected in the negative feedback fashion. Each population was described by the time courses of postsynaptic potentials and sigmoid transfer functions, which were used to simulate the conversion between the mean membrane potential of a neuronal population and the density of firing of the population. The interaction between the two populations was controlled by the coupling constants representing the average number of synaptic contacts between the two cell types. White Gaussian noise was fed into the network by means of an excitatory input to the TCR population. The output of the model was the average of the postsynaptic potentials of the TCR cells and it simulated what is generally measured by recording local field potentials or EEG signals. For a physiological range of model parameters the output signal exhibited oscillations of frequency $8-11 \mathrm{~Hz}$ closely resembling cortical alpha rhythm. The linear system analysis revealed that the power spectra obtained for increasing values of coupling strength between the populations displayed a progressive change from predominantly low pass characteristic to a clear band pass spectrum. The family of spectra closely approximated the development of the EEG as a function of age. In this way system analysis of the alpha rhythm model provided a hypothesis that the evolution of the posterior EEG rhythm from the low dominant frequency to the alpha rhythm signal having a clear peak at around $11 \mathrm{~Hz}$ would depend upon an increase in number of interconnections and efficiency of synaptic contacts. The results of the linear analysis led to a general conclusion that neural networks act as band pass linear filters and the resulting output signals, such as alpha rhythmic activity observed in EEG, may be described, in a first approximation, as linearly filtered noise. Due to its simplicity and generality this alpha rhythm model became a model for the generation of rhythmic activities and it has been adapted by many successive studies ([122] - [129]).

\section{SYSTEM LEVEL/GLOBAL MODELS}

\section{A. Nunez's cortical model}

Nunez's mathematical approach of neocortical dynamics led to the development of global theories ([130], [131]) and combinations of global and local theories ([132], [133]). Local circuits include positive and negative feedback loops at millimeter scales, where signal delays are determined mostly by postsynaptic potential rise and decay times. The global approach neglects local delays but takes into account propagation delays along cortico-cortical fibres that provide positive feedback between multiple cortical regions. In this way the model can predict dominant oscillation frequencies in the general range of observed EEG phenomena in humans. The global oscillations are influenced by the boundary conditions that are determined by the size and geometry of neocortex and cortico-cortical connections. Global theory predicts, among others, a correlation between alpha frequency and head size. Local circuits generate higher frequency oscillations that propagate between neocortical columns. Some principal results of Nunez's theory have been confirmed experimentally ([134], [135]). 


\section{B. Wright's cortical and thalamocortical models}

The models of the Wright's group are large scale models of electrical activity of the brain that provide a unified description of the main EEG rhythms using realistic neurophysiological parameters. In the spatially discrete model ([136]) the cortex was approximated by a matrix of unit volumes. Each unit volume consisted of two masses, excitatory and inhibitory ones, connected to each other and recurrently to themselves. Connections within a unit volume included dendritic delays related to rise and decay times of postsynaptic potentials. The connections between the units accounted for cortico - cortical couplings: their strength diminished with distance while the delay was proportional to the separation between the units. In each mass, the local field potential and the pulse density were related by a sigmoidal function. The major features of these models include: peaks in the power spectrum of the output signals at the frequencies corresponding to main EEG rhythms (theta, alpha, beta and gamma), a general ' $1 / \mathrm{f}$ ' decrease of spectral power as a function of frequency and an increase of output peak frequency as a function of non-specific activation (a constant excitatory input applied to all matrix elements). The frequency - wavenumber estimates together with phase velocities and spatial attenuation of traveling waves present at the steady state were in good agreement with physiological measurements. Additionally, the simulations of the responses of visual stimulation with moving bars (simulated as a set of cross-correlated inputs) gave good agreement with experimental observations of spatially synchronous oscillations in the gamma frequency range ([137]). The continuous form of the Wright's model was introduced by Robinson [138]. Using the set of continuous nonlinear equations describing the waves of cortical electrical activity, a steady - state and stability analysis was performed and dispersion relation for linear waves were obtained analytically. This continuous formulation was also more tractable to study the effects of boundary conditions exerted on global frequencies for different cortical geometries. The feedback cortex - thalamus has been subsequently added to the model ([139], [140]). System impulse responses were used to model the brain's transient responses to discrete stimuli allowing for investigation of the neurophysical basis of evoked potentials. The extended corticothalamic (i.e. involving both the cortex and thalamus) model also allowed to simulate a wide range of spectral peaks observed experimentally under non pathological conditions (slow wave, delta, theta, alpha and sleep spindles) as well as nonlinear behavior (limit cycle) corresponding to epilpetiform EEG during epileptic seizures ([141]).

An interesting attempt to bridge the microscopic cellular and macroscopic EEG models of neocortical dynamics was undertaken by Wright and Liley ([142]). At the scale of single cells the dynamics is characterized by nonlinear equations, whereas processes at the EEG level exhibit linear and nearequilibrium dynamics. One of the authors' conclusion is that the microscopic complex, possibly chaotic, behavior is compatible with almost linear behavior describing neural mass dynamics due to spatially and temporally noisy input from the reticular formation. Although this article doesn't fully explore the multiscale modeling of the brain, it brings up some fundamental questions in computational neuroscience and it became a subject of open peer commentary contributed by many experts in the field.

\section{MODELS OF EPILEPTIC ACTIVITY}

Epilepsy is a complex dynamical disease characterized by recurrent seizures. About 0.5 to $1 \%$ of the population is affected by epilepsy which is, next to stroke, the most common neurological disease. Although there is currently strong evidence that seizures are related to abnormal excessive firing and synchronization of neurons in a part of the brain, little is known about the precise basic mechanisms underlying human epileptic seizures and mechanisms of transitions from normal to epileptic activity. The review article of Lopes da Silva [143] presents three different routes of transitions between normal, seizure - free state and the state characterized by epileptic seizures. The first route, called 'attractor deformation', consists of gradual changes of network parameters leading to smooth and continuous changes of system behavior from interictal (before seizure) to ictal (seizure) activity. In an alternative, 'bifurcation' route, the system features two operational states, normal and paroxysmal, that may coexist for the same sets of parameters. Whether one or the other are present depends on external inputs or internal random perturbations of the system; the latter may lead to a sharp transition from normal to paroxysmal behavior. The third, 'mixed' scenario is a mixture of the two basic scenarios in which gradual deformation of the operational normal state facilitates the sharp transition to the ictal state. As discussed in [143] the identification of dynamical processes leading to seizure generation may have a fundamental impact on the ability to predict seizure occurrence and on the development of new therapeutic interventions (e.g., deep brain stimulation). Here, we present two computational models at the population level that cover the two main scenarios of seizure generation and correspond to two different epileptic phenomena: (i) partial seizures occurring in temporal lobe epilepsy and (ii) generalized absence seizures.

\section{A. Human Partial epilepsy}

In this section, we present a modeling attempt to relate electrophysiological patterns of epileptic activity to pathophysiological mechanisms involved during the transition to seizure in a specific form of human partial epilepsy called temporal lobe epilepsy (TLE). In TLE, seizures originate in one or several anatomic areas of the temporal lobe and propagate through interconnected neuronal networks within or beyond its boundaries. Medial temporal lobe epilepsy (MTLE) is the first and most common subtype of TLE. It has been extensively studied over the last decade resulting in the description of the so called "MTLE syndrome" ([144], [145]) 
Fig. 3 over here

characterized by a significant volume reduction of certain medial (limbic) structures, particularly the hippocampus. Drug treatment is often inefficient in patients with TLE and surgical excision of the epileptogenic region and/or seizure propagation pathways remain the only way to stop or significantly reduce the frequency of seizures. However, the rate of surgical failure in this population is far from negligible ([146]), suggesting that the understanding of seizuretriggering mechanisms still constitutes an essential step for improving the surgical outcome or for developing new therapeutic strategies like electrical stimulations. During presurgical evaluation of patients with intractable TLE, intracerebral recordings (depth-EEG) can be performed. In this context, the general objective of this work was to make use of physiologically-relevant models of neuronal populations to interpret depth-EEG signals recorded during the transition from interictal (before seizure) to ictal (seizure) activity.

Modeling at the neuronal population level was chosen for two main reasons. First, it appeared well suited to the nature of our real observations. Indeed, macroelectrodes which are used for depth-EEG recording provide signals arising from large assemblies of cells (field activity). Second, excitation- or inhibition-related parameters are considered as essential in the study of epileptic processes. Neuronal population models include parameters directly related to excitation, inhibition or couplings between neuronal subpopulations present in explored brain structures.

Starting from aforementioned works (part V), a canonical form of this class of models (Fig. 3a) representing a cluster of neurons containing three interacting subsets was studied ([125]). The first subset is composed of the main cells (i.e. pyramidal cells in the hippocampus or neocortex). It receives feedback from the two other subsets composed of local inhibitory interneurons and other excitatory cells. Taking into account some hypotheses about epileptic mechanisms related to imbalance between excitation and inhibition, model parameters (related to the amplitude of average excitatory or inhibitory post-synaptic potentials) were altered; accordingly epileptiform signals could be simulated, the dynamics of which strongly resemble those of signals recorded from different brain structures for both interictal and ictal activities. However, it was also observed that the model was not able to represent fast EEG activities such as rapid discharges often observed in intracerebral recordings at seizure onset. This inconsistency pointed towards necessary model improvement.

A new design for the model of hippocampal activity was then proposed, starting from basic neuroscientific studies about i) the role of inhibitory interneurons in hippocampal or neocortical networks in the generation of gamma frequency oscillations ([69], [147]), ii) the non-uniform alteration of GABAergic inhibition in experimental models of temporal epilepsy (reduced dendritic inhibition and increased somatic inhibition) ([148]), and iii) the possible depression of
$\mathrm{GABA}_{\mathrm{A}, \text { fast }}$ circuit activity by $\mathrm{GABA}_{\mathrm{A}, \mathrm{slow}}$ inhibitory postsynaptic currents in the hippocampus ([149]). These studies highlighted the necessity to consider not only one but two inhibitory subpopulations in the model in order to take into account two separate classes of interneurons (called, for simplicity, $\mathrm{GABA}_{\mathrm{A}, \text { fast }}$ interneurons and $\mathrm{GABA}_{\mathrm{A} \text {,slow }}$ interneurons) that were demonstrated to give rise to slow and fast inhibitory postsynaptic currents (IPSCs). The new model was designed to represent this cellular organization of interacting subsets of principal neurons and interneurons, as summarized in Fig. 3b. It consists of three subsets of neurons, namely the main cells (i.e. pyramidal cells), the slow dendritic-projecting inhibitory interneurons $\left(\mathrm{GABA}_{\mathrm{A} \text {,slow }}\right.$ receptors) and the fast somatic-projecting inhibitory interneurons $\left(\mathrm{GABA}_{\mathrm{A}, \mathrm{fast}}\right.$ receptors). Three key model parameters are directly related to excitatory, slow inhibitory and fast inhibitory synaptic interactions between subsets of cells. These three parameters are referred to as EXC, SDI and FSI, respectively.

In a first step, the model was shown to produce strikingly realistic activities when compared to real depth-EEG signals (Fig. 3c). In a second step, it was used to study and interpret the transition from background to seizure activity (see example in Fig. 4a), in term of the evolution of EXC, SDI and FSI parameters. Thus, a parameter sensitivity analysis was carried out ([126]) using an exhaustive procedure aimed at uncovering, from simulations, disjoint regions in the space of free parameters, each region being associated to a particular type of model activity. Then, activities reflected in real signals as well as transitions between activities were interpreted as possible paths connecting corresponding regions in the space of free parameters. As this space is of dimension 3, paths were displayed in the (SDI, FSI) plane, for different values of EXC. (Fig. 4b). Results demonstrated that the transition from interictal to ictal activity can be explained by time-varying synaptic interactions between pyramidal cells and interneurons with slow and fast GABAa kinetics. In particular, the model explained the appearance of the rapid discharge at seizure onset by a drop of dendritic inhibition. It also permitted to relate the observed fast oscillations to the crucial role of interneurons projecting to the somatic region of pyramidal cells.

In summary, results showed that neuronal population models can be adapted to specific cerebral structures like the hippocampus by integration of available neuronal histological data and can produce realistic epileptiform activities and transitions between activities (Fig. 4d and Fig. 4e). This computational modeling study offered the unique opportunity to relate electrophysiological patterns typically recorded with intracerebral electrodes during the transition from interictal to ictal activity in the human hippocampus to ictogenesis mechanisms and to generate hypotheses about these mechanisms.

\section{B. Non - convulsive epilepsy}

In this section a computational model of absence seizures, 
that are assumed to involve thalamocortical circuits, is

Fig. 4 over here

presented. Such seizures are paroxysmal losses of consciousness that start and end abruptly and are accompanied by bilaterally synchronous rhythmic spike and wave (SW) discharges that can be recorded on the EEG. Experimental findings and computational models ([107], [108], [150]) have given insight into some basic neuronal mechanisms of SW discharges. However the mechanisms that are responsible for the spontaneous transition between the normal and paroxysmal SW activity are not well understood. Similarly, the questions of thalamocortical versus cortical mechanisms of SW generation are not fully resolved.

These questions were approached using a thalamocortical computational model ([128]). The model was constructed at the intermediate level between the distributed neuronal network and lumped circuit levels. That is, the explicit behavior of individual neurons was not simulated but rather the network of interacting populations was considered. In neuronal populations, specific mechanisms at the neurons" membrane level were included. Such approach involves a number of simplifications of the real system but exhibits a number of advantages. First, using a relatively simple model which is able to replicate specific experimental results, one can define a basic set of mechanisms/rules that are necessary and sufficient to account for the particular observed phenomena. Second, this approach enables to investigate system dynamics at the macroscopic level, that is at the level where electric brain signals such as local field potentials, or EEG, are recorded. Overall system dynamics is usually hardly accessible in distributed neuronal models. Third, such model is computationally efficient and allows to simulate long time range ( hours or days) behavior.

\section{Fig. 5 over here}

The model is based on physiology of the thalamocortical network shown schematically in Fig. 5a. It represents an extension of the lumped model alpha rhythm model [121] described in section V.C. The schematic diagram of the model is shown in Fig. 5b. The model consists of two loops, cortical and thalamic ones, that are mutually interconnected. The thalamic loop is formed by a population of thalamocortical (TC) cells that projects to a population of reticular thalamic (RE) cells. The latter inhibits TC population by way of $\mathrm{GABA}_{\mathrm{A}}$ and $\mathrm{GABA}_{\mathrm{B}}$ types of inhibition. The TC cells receive external excitatory input that represents sensory inputs from the ascending afferents while the RE population receives external inhibitory input. The latter represents the input from the neighbouring RE cells, since the latter are interconnected by mutual inhibitory synapses. The cortical circuit consists of a negative feedback loop formed by interacting populations of pyramidal cells (PY) and inhibitory interneurons (IN). Pyramidal cells, in addition to projecting to interneurons, send also excitatory connections to the thalamus both to the TC and
RE populations. In turn, the TC cells excite both the pyramidal cells and interneurons. The PY population receives also external cortical input that stands for the excitatory input from other pyramidal cells, not included in the lump. All excitatory synaptic interactions are mediated by glutamatergic AMPA receptors. Thalamic populations receive also modulating inputs corresponding, among others, to cholinergic activation from the brain stem. The output of the model, is the mean membrane potential of the pyramidal cell population.

The main results obtained through model simulations and system analysis were the following: (i) Paroxysmal discharges characterised by $9 \mathrm{~Hz}$ large amplitude oscillations arose and ceased spontaneously during model operation, without any changes of model parameters (Fig. 5c). (ii) Bifurcation analysis of the model showed that the network exhibits bistable dynamics, i.e. the normal and paroxysmal state coexist for the same set of parameters. Noise present in the network may switch the system between these two types of behavior. (iii) The distributions of lengths of ictal and seizure free epochs were exponential (Fig. 5d), indicating that transitions between the two stable states occurred randomly over time. This model prediction was validated using experimental data from animal models of absence epilepsy, that display SW at about $9 \mathrm{~Hz}$, and human subjects ([151]). (iv) Variation of model's parameters has shown that depending on the parameter setting, the paroxysmal oscillation may be generated purely thalamically, purely cortically or in interacting thalamocortical network. This result could partly reconcile the seemingly contradictory experimental observations of SW originating primarily in the cortex or in the thalamus ([152]). (v) Since the sudden onset of large amplitude paroxysmal activity is triggered by a stochastic process, this implies that the occurrence of this type of seizures is unpredictable per definition. (vi) Model simulations confirmed theoretical predictions that in bistable systems the large amplitude oscillation may be annihilated by a single well - timed pulse. This pointed to the possibility of arresting seizures having certain dynamical properties. In summary, a simple computational model constructed with relatively low complexity provided interpretation of experimental findings, novel testable hypotheses, suggestions for new experiments and new insights of clinical importance.

\section{Implications for understanding of epileptic brain activity}

The studies presented in this chapter were motivated by clinical questions and dealt with pathological brain behavior observed during epileptic seizures. Both models were constructed at the same (population) scale and were based on physiological and clinically relevant data (depth-EEG and scalp-EEG). They provided novel insights concerning the mechanisms of transistions from normal to seizure activity in two different types epileptic phenomena (in terms of clinical manifestations and networks involved). The model of the hippocampal network activity described in section VII.A 
predicted a sequence of changes in parameters determining synaptic interactions between subpopulations of (inter)neurons that can lead to seizure generation. The computational model of absence epileptic process described in section VII.B showed that some classes of epileptic behavior could be characterized by bistability properties. In this case, seizure generation doesn't require any parameter change and can be induced by unpredictable stochastic fluctuations, which are inherent to any biological system. These studies were described in more detail here since they provide examples of how interaction between computer modelers, experimentalists and clinicians may contribute to significant advances in understanding of complex brain pathology. These two models also illustrate that one cannot develop a general computational model of epilepsy. The diversity of epilepsies requires construction of specialized models carefully adapted to particular epilepsy type.

\section{TECHNOLOGY OF BRAIN MODELING}

\section{A. Modeling software}

Numerous software packages are available to build accurate models of single neurons and networks of neurons and perform simulations. Most of them are based on compartmental modeling which introduces a spatial discretization of neurons to simplify the equations describing, in time and space, the dynamics of ion-specific channels, the synaptic interactions between cells and propagation aspects of membrane potentials. These packages can be used to accurately represent the various neuronal morphologies encountered in a given real neuronal tissue. They mainly differ in the organization of the user interface (presenting the modeler with an intuitive environment in which details of the numerical methods are hidden), in the level of physiological detail that can be added to model neurons and in the way integration methods are implemented.

Detailed neuron models may now have more than one thousand compartments with multiple ionic conductances. At the cell level, some packages can handle this complexity. However, for network simulations, supercomputers and parallel-processing are still required, the alternative being the use of simplified neuron models in large networks. In most packages, standard numerical methods are available to solve the set of differential equations that govern a given neuron model (like forward, backward or exponential Euler methods). In some packages e.g., Neuron ([153]) and Genesis ([154]), a method due to Hines ([155]) is available. It provides an efficient way to solve these equations and may be used to speed up simulations in the case where the coupled equations describe a branching tree-like structure (such as the dendrites of a neuron) without closed loops. This brief paragraph only gives a short account of the available neuronal modeling software. Although written more than 10 years ago, the comparative review by de Schutter [156] stays very informative for readers who wish more details about the functionalities of these software packages. Recently, new computer languages are being developed for description and simulation of neural systems, e.g., NSL - Neural Simulation Language ([157]). Storage and exchange of computer-based mathematical models is facilitated by the CellML language ([158]). It allows scientists to share models even if they are using different model-building software.

\section{B. Neuron - silicon interface}

Apart from simulation environments dedicated to modeling neural systems various techniques have been developed that integrate real biological neurons with computer-simulated or electronic chips environment. The dynamic clamp uses computer simulation to introduce artificial membrane or synaptic conductances into biological neurons and to create networks from previously unconnected neurons or hybrid circuits of real and model neurons. In this approach, it is essential that models can run in real time, which has become possible with the current generation of computers. The review article ([159]) describes recent high-tech implementations of this technique and summarizes insights gained through its use. The integration of individual nerve cells, small neuronal networks or even brain slices with microelectronic circuits is a new way to explore complex neural processes ([160]). Possible applications of this approach in brain research, biosensorics, information technology and medical prosthetics are reviewed in [161].

\section{Neuromorphic engineering}

Designing electronic devices mimicking real neuronal operations in the brain is a new branch of engineering. Douglas and coworkers ([162]) designed an analog VLSI chip that matches the properties of real biological neurons including continuous dendritic and synaptic computations. A single - chip model system of signal processing in the auditory brainstem was presented in [163]. A multi-chip VLSI neuronal system approximating a cortical microcircuit has been subsequently developed to model visual processing performed by orientation - tuned neurons ([164]). Such an analog hardware system has advantages over computer neuronal models in that it is very efficient computationally and the computational time does not scale with the size of the neuronal network. An extended model of the visual system based on the same technology was presented in [165]. The realistic behavior of the silicon system suggested the possibility of implementation of similar circuits for visual prosthetics. The largest neuromorphic system built to date was an artificial organism 'Ada' that integrated a variety of sensory and behavioral modalities ([166]).

\section{DISCUSSION}

Computational neuroscience is a relatively young branch of science and it is at present in a fast growing phase as compared to general neuroscience, which grows also strongly but more steadily, as quantified by a number of publications per year (Fig. 6). The proliferation of brain models can be explained on the one hand simply by and the growing 
availability of fast digital computers, and on the other, it may indicate increasing awareness of, and confidence in, theoretical approaches based on computational models. An increasing demand for the integration of experimental and theoretical approaches in the neurosciences is becoming more common. Indeed, a number of studies presented in this review were developed as an attempt to provide insight and to put into a common framework a vast amount of experimental, sometimes apparently contradictory, findings. Some concepts relevant to understand brain functions could not have been even reached based only on experimental observations alone. E.g., Prinz [167] by simulating 20 million versions of the model using different connection strengths and neuron properties showed that a wide heterogeneity in parameters may still lead to the same network activity. This may be central to understand biological systems that always exhibit some degree of variability. Other models provided insight into how the modification of system parameters, or intrinsic fluctuations, may lead to abnormal behavior, such as the transition between normal activity and epileptic seizures. These results obtained using a modeling approach corroborate experimental results and make some predictions concerning clinically relevant questions like seizures predictability or seizure abortion. These studies are significant examples of how modeler - experimentalist dialogues may lead to progress in this field.. Ways to improve this interdisciplinary interaction have been also suggested in [168].

Another important issue concerns the question of scale i.e., the level of neuronal organization at which the model should be set. All models presented in this review integrate and "organize" some knowledge at a given scale. Most of them are intimately linked to experiments. They are used to test hypotheses on available data or to relate observed phenomena to the underlying mechanisms. New experimental data should lead to model adjustments or to the development of new models. In turn, models may be helpful in the design of new experiments. However, too detailed models may be too complex to analyse and interpret, while too general models may not capture the essence of some experimental phenomenon. The modeling level should be appropriately chosen to match the neurophysiological level of the experimental observations that are to be explained. E.g., the compartmental modeling level was appropriate to gain insight into the burst generation occurring at the soma as well as in the dendrites of the CA3 cells ([52]) but turned out to be not necessary to explain the generation of intrinsic bursts in thalamic relay cells ([56]). Similarly, evoked potentials can be easily simulated using population models ([124], [140]) while this approach would probably fail to capture memory processes, which depend on modifications in the strengths of synaptic connections between individual cells ([37]).

Apart from physiologically based models, abstract models are also being developed that consider the brain as a physical dynamical system without detailed assumptions about the elements that constitute it ([169] - [172]). Such models although not providing direct insight into neurophysiological mechanisms can provide a common conceptual framework and may reveal unifying principles, by showing e.g., that various seemingly different complex phenomena are governed by the same simple rules.

Although progress in understanding brain functions is significant, we are still far from achieving this goal. Many neuroscientists are becoming aware that understanding neural processes requires the development of brain models at the global and local scales. Indeed, an essential feature at one level might be an insignificant detail at another level and no single model can be expected to span all the levels of organization briefly described in this review. Although first attempts to build a unified theory that integrate different levels of neuronal organization have already been made (Wright and Liley, 1986; Nunez, 1989, 1995), a challenge to develop computational brain models that bridge microscopic and macroscopic levels is still facing modelers.

\section{REFERENCES}

[1] G. M. Shepherd, Neurobiology. New York: Oxford University Press, 1994.

[2] L. J. Graham "Biophysical mechanisms in neuronal modeling," in The Handbook of Brain Theory and Neural Networks, 2nd ed. M. A. Arbib, Ed., Cambridge, MA, MIT Press, 2003.

[3] W. S. McCulloch, W. Pitts, "A logical calculus of the ideas immanent in nervous activity,” Bulletin of Mathematical Biophysics, vol. 5, pp. 115133, 1943.

[4] A. L. Hodgkin, W. A. H. Rushton, "The electrical constants of a crustacean nerve fibre," Proc. R. Soc. Lond., vol. 133, pp. 444-479, 1938.

[5] J. S. Coombs, J. C. Eccles, P. Fatt, "The electrical properties of the motoneurone membrane,” J. Physiol. vol. 130, pp. 291-325, 1955.

[6] J. S. Coombs, J. C. Eccles, P. Fatt, "The specific ionic conductances and the ionic movements across the motoneurone membrane that produce the inhibitory post-synaptic potential,” J. Physiol., vol. 130, pp. 326-373, 1955.

[7] W. Rall, "Membrane time constants of motoneurons," Science, vol. 126, pp. 454, 1957.

[8] W. Rall, "Branching dendritic trees and motoneuron membrane resistivity,” Exp. Neurol., vol. 1, pp. 491-527, 1959.

[9] W. Rall, "Time constants and electrotonic length of membrane cylinders and neurons,” Bioph. J., vol. 9, pp. 1483-1508, 1969.

[10] W. Rall, "Electrophysiology of a dendritic neuron model," Bioph. J., vol. 2, pp. 145-167 1962.

[11] W. Rall, Theory of physiological properties of dendrites. Ann. N. Y. Acad. Sci., vol. 96, 1071-1092, 1962.

[12] A.L. Hodgkin and B. Katz, " The effect of temperature on the electrical activity of the giant axon of the squid,” J. Physiol., vol. 109, pp. 240249, 1949.

[13] A. L. Huxley and A. F. Hodgkin, "A quantitative description of membrane current and its application to conduction and excitiation in nerve,” J. Physiol., vol. 1, pp. 500-544, 1952e.

[14] R. FitzHugh, "Impulses and physiological states in models of nerve membrane,” Biophys. J., vol. 1, pp. 445-466, 1961.

[15] S. Nagumo, S. Arimoto and S. Yoshizawa, "An active pulse transmission line simulating nerve axon,” Proc. IRE, vol. 50, pp. 20612070, 1962.

[16] Morris and H. Lecar, "Voltage oscillations in the barnacle giant muscle fiber,” Biophys. J., vol. 35, pp. 193-213, 1981.

[17] W. Gerstner and W. M. Kistler, Spiking Neuron Models: Single Neurons, Populations, Plasticity, Cambridge: Cambridge University Press, 2002.

[18] J. Wu, "Microscopic model for selective permeation in ion channels," Biophys. J., vol. 60, pp. 238-251, 1991. 
[19] O. Beckstein and M. S. P. Sansom, "Liquid-vapor oscillations of water in hydrophobic nanopores”. Proc. Nat. Acad. Sci. vol. 100, pp. 70637068, 2003.

[20] S. S., Deol, P. J. Bond, C. Domene and M. S. P. Sansom, "Lipid-protein interactions of integral membrane proteins: a comparative simulation study,” Biophys. J. vol. 87, pp.3737-3749, 2004.

[21] P. Tang and Y. Xu, "Large-scale molecular dynamics simulations of general anesthetic effects on the ion channel in the fully hydrated membrane: The implication of molecular mechanisms of general anesthesia,” Proc. Nat. Acad. Sci., vol. 99 (25), pp. 16035-16040, 2002.

[22] B. C. Chow and J. A. White, "Spontaneous action potentials due to channel fluctuations,” Biophys. J., vol. 71, pp.3013-3021, 1996.

[23] A. M. Colbert and E. H. Pan, "Ion channel properties underlying axonal action potential initiation in pyramidal neurons," Nature Neurosci., vol. 5, pp. 533-538, 2002.

[24] Z. F. Mainen, J. Joerges, J. R. Huguenard, T. J. Sejnowski, “A model of spike initiation in neocortical pyramidal neurons” Neuron, vol., 15, pp. 1427-1439, 1995.

[25] T. B. Kepler, E. Marder, "Spike initiation and propagation on axons with slow inward currents,” Biol. Cybern., vol. 68, pp. 209-214, 1993.

[26] L. Cathala, S. Brickley, S. Cull-Candy and M. Farrant, "Maturation of EPSCs and intrinsic membrane properties enhances precision at a cerebellar synapse,” J. Neurosci., vol. 23, pp. 6074-6085, 2003.

[27] R. Carbunaru and D. M. Durand, "Axonal stimulation under MRI magnetic field z gradients: a modeling study,” Magnetic Resonance in Medicine, vol. 38, pp. 750-758, 1997.

[28] A. Destexhe, Z. Mainen, T. J. Sejnowski, “An efficient method for computing synaptic conductances based on a kinetic model of receptor binding," Neural Comput., vol. 6, pp.14-18, 1994.

[29] A. Destexhe, Z. F. Mainen and T. J. Sejnowski, "Biophysical models of synaptic transmission,” in Methods in Neuronal Modeling, 2nd ed., C. Koch and I. Segev, I. Ed. Cambridge: MIT press, 1998, pp. 1-25.

[30] A. Destexhe, M. Rudolph, J. M. Fellous, T. J.Sejnowski, "Fluctuating synaptic conductances recreate in vivo-like activity in neocortical neurons,” Neuroscience, vol. 107, pp. 13-24, 2001.

[31] W. Maass and A. Zador, "Dynamic stochastic synapses as computational units,” Neural Comput., vol. 11, pp. 903-917, 1999.

[32] H. D. Abarbanel, R. Huerta, M. I. Rabinovich, "Dynamical model of long-term synaptic plasticity,” Proc. Nat. Acad. Sci. vol. 99, pp. 1013210137, 2002.

[33] S. Fusi, M. Annunziato, D. Badoni, A. Salamon, D. J. Amit, “Spikedriven synaptic plasticity: theory, simulation, VLSI implementation,” Neural Comput., vol. 12, pp. 2227-2258, 2000.

[34] J. Lisman, “A mechanism for Hebb and anti-Hebb processes underlying learning and memory,” Proc. Nal. Acad. Sci., vol. 86, pp. 9574-9578, 1989.

[35] A. Zador, C. Koch and T. H. Brown, "Biophysical model of a hebbian synapse,” Proc. Nat. Acad. Sci., vol. 87, pp. 6718-6722, 1990.

[36] J. I. Gold and M. F. Bear, "A model of dendritic spike $\mathrm{Ca}^{2+}$ concentration exploring possible basis for sliding synaptic modification threshold," Proc. Nat. Acad. Sci., vol. 91, pp.3941-3945, 1994.

[37] H. Z. Shouval, M. F. Bear and L. N. Cooper, “A unified model of calcium dependent synaptic plasticity,” Proc. Nat. Acad. Sci., vol. 99(16), pp. 10831-10836, 2002.

[38] L. C. Yeung, H. Z. Shouval, B. S. Blais, L. N. Cooper, "Synaptic homeostasis and input selectivity follow from a calcium-dependent plasticity model,” Proc. Nat. Acad. Sci., vol. 101(41), pp. 14943-14948, 2004.

[39] E. Marder, "From biophysics to models of network function," Annu. Rev. Neurosci., vol. 21, pp. 25-45, 1998.

[40] A. Destexhe and E. Marder, "Plasticity in single neuron and circuit computations,” Nature, vol. 431, pp.789-95, 2004

[41] M. C. Wiest, D. M. Eagleman, R. D. King and P. R. Montague, "Dendritic spikes and their influence on extracellular calcium signalling,” J. Neurophysiol., vol. 83, pp. 1329-1337, 2000.

[42] R. D. King, M. C. Wiest and P. R. Montague, "Extracellular calcium depletion as a mechanism of short-term synaptic depression," $J$. Neurophysiol., vol. 85, pp. 1952-1959, 2001.

[43] H. Kager, W. J. Wadman, G. G. Somjen, "Simulated seizures and spreading depression in a neuron model incorporating interstitial space and ion concentrations,” J. Neurophysiol., vol. 84, pp. 495-512, 2000.
[44] H. Kager, W. J. Wadman, G. G. Somjen, "Conditions for the triggering of spreading depression studied with computer simulations," $J$. Neurophysiol., vol. 88, pp. 2700-2712, 2002.

[45] B. E. Shapiro, "Osmotic forces and gap junctions in spreading depression: a computational model,” J. Comput. Neurosci., vol. 10, pp. 99-120, 2001.

[46] C. Bedard, H. Kroger and A. Destexhe, "Modeling extracellular field potentials and the frequency-filtering properties of extracellular space," Biophys. J., vol. 86, pp. 1829-1842, 2004.

[47] M. Martina, I. Vida and P. Jonas, "Distal initiation and active propagation of action potentials in interneuron dendrites,” Science, vol. 287, pp. 295-300, 2000.

[48] W. Rall, G. M. Shepherd, "Theoretical reconstruction of field potentials and dendrodendritic synaptic interactions in olfactory bulb," $J$. Neurophysiol., vol. 31, pp. 884-915, 1968.

[49] F. A. Dodge and J. W. Cooley, "Action potential of the motorneuron.” IBM Journal of Research and Development, vol. 17, pp. 219-229, 1973.

[50] F. A. Dodge, "The nonuniform excitability of central neurons as exemplified by a model of the spinal motoneuron," in The Neurosciences: Fourth Study Program, F. O. Schmidt and F. G. Worden, Ed. Cambridge, MA: MIT Press, 1979, pp. 439-455.

[51] R. D. Traub, "Neocortical pyramidal cells: a model with dendritic calcium conductance reproduces repetitive firing and epileptic behavior,” Brain Res., vol. 173(2), pp. 243-257, 1979.

[52] R. D. Traub, "Simulation of intrinsic bursting in CA3 hippocampal neurons,” Neuroscience, vol. 7(5), pp. 1233-1242, 1982.

[53] R. D. Traub, R. Llinas, "Hippocampal pyramidal cells: significance of dendritic ionic conductances for neuronal function and epileptogenesis," J. Neurophysiol., vol. 42(2), pp. 476-496, 1979.

[54] R. D. Traub, E. H. Buhl, T. Glovell and M. A. Whittington, "Fast rhythmic bursting can be induced in layer 2/3 cortical neurons by enhancing persistent $\mathrm{Na}+$ conductance and blocking BK channels," $J$. Neurophysiol., vol. 89, pp. 909-921, 2003.

[55] J. R. Huguenard, D. A. McCormick, "Simulation of the currents involved in rhythmic oscillations in thalamic relay neurons," $J$. Neurophysiol., vol. 68, pp. 1373-1383, 1992.

[56] A. McCormick, J. R. Huguenard, “A model of the electrophysiological properties of thalamocortical relay neurons” J. Neurophysiol., vol. 68, pp.1384-1400, 1992.

[57] X. J. Wang, "Multiple dynamical modes of thalamic relay neurons: rhythmic bursting and intermittent phase-locking," Neuroscience, vol. 59(1), 21-31, 1994

[58] A. Destexhe, D.Contreras, M. Steriade, T. J. Sejnowski, J. R. Huguenard, "In vivo, in vitro, and computational analysis of dendritic calcium currents in thalamic reticular neurons," J. Neurosci., vol. 16, pp. 169-185, 1996

[59] J. M. Fellous, M. Rudolph, A. Destexhe, T. J. Sejnowski, "Synaptic background noise controls the input/output characteristics of single cells in an in vitro model of in vivo activity," Neuroscience, vol. 122, pp. 811829, 2003.

[60] F. Saraga, C. P. Wu, L. Zhang and F. K. Skinner, “Active dendrites and spike propagation in multi-compartment models of orienslacunosum/moleculare hippocampal interneurons,” J. Physiol., vol. 552, pp. 673-689, 2003.

[61] I. Segev and M. London, "Untangling dendrites with quantitative models” Science, vol. 290, pp. 744-750, 2000.

[62] E. De Schutter, "Using realistic models to study synaptic integration in cerebellar Purkinje cells,” Rev. Neurosci., vol.10, pp. 233-245, 1999.

[63] D. K. Hartline, A. M. Castelfranco, "Simulations of voltage clamping poorly space-clamped voltage-dependent conductances in a uniform cylindrical neurite,” J. Comput. Neurosci., vol. 14, pp. 253-269, 2003.

[64] A. M. Castelfranco, D. K. Hartline, "Corrections for space-clamp errors in measured parameters of voltage-dependent conductances in a cylindrical neurite,” Biol. Cybern., vol. 90, pp. 280-290, 2004.

[65] R.D. Traub, R. Miles and R.K. Wong, "Model of the origin of rhythmic population oscillations in the hippocampal slice,” Science, vol. 243, pp. 1319-1325, 1989.

[66] R. D. Traub and R. Dingledine, "Model of synchronized epileptiform bursts induced by high potassium in CA3 region of rat hippocampal slice. Role of spontaneous EPSPs in initiation,” J. Neurophysiol., vol. 64, pp. 1009-1018, 1990 
[67] R. D. Traub, R. Miles and G. Buzsaki, "Computer simulation of carbachol-driven rhythmic population oscillations in the CA3 region of the in vitro rat hippocampus,” J. Physiol., vol. 451, pp. 653-672, 1992.

[68] P. F. Pinsky and J. Rinzel, "Intrinsic and network rhythmogenesis in a reduced Traub model for CA3 neurons," J. Comput. Neurosci., vol. 1, pp. 39-60, 1994.

[69] J. G. Jefferys, R. D. Traub, M. A. Whittington, "Neuronal networks for induced '40 Hz' rhythms," Trends Neurosci., vol. 19, pp. 202-208, 1996.

[70] R. D. Traub, M. A. Whittington, I. M. Stanford, J. G. Jefferys, “A mechanism for generation of long-range synchronous fast oscillations in the cortex," Nature, vol. 383(6601), pp. 621-624, 1996.

[71] Rinzel, D. Terman, X. Wang, B. Ermentrout, "Propagating activity patterns in large-scale inhibitory neuronal networks,” Science, vol. 279(5355), pp. 1351-1355, 1998.

[72] R. D. Traub, A. Bibbig, "A model of high-frequency ripples in the hippocampus based on synaptic coupling plus axon-axon gap junctions between pyramidal neurons," J. Neurosci., vol. 20(6), pp. 2086-2093, 2000.

[73] R. D. Traub, I. Pais, A. Bibbig, F. E. LeBeau, E. H. Buhl, S. G. Hormuzdi, H. Monyer, M. A. Whittington, "Contrasting roles of axonal (pyramidal cell) and dendritic (interneuron) electrical coupling in the generation of neuronal network oscillations,” Proc. Nat. Acad. Sci., vol. 100(3), pp. 1370-1374, 2003.

[74] P. J. Franaszczuk, P. Kudela, G. K. Bergey, "External excitatory stimuli can terminate bursting in neural network models," Epilepsy Res., vol. 53, pp. 65-80, 2003.

[75] K. H. Yang, P. J. Franaszczuk, G. K. Bergey, "Inhibition modifies the effects of slow calcium-activated potassium channels on epileptiform activity in a neuronal network model," Biol. Cybern., vol. 92(2), pp. 7181, 2005.

[76] A. Compte, M. V. Sanchez-Vives, D. A. McCormick, X. J. Wang, "Cellular and network mechanisms of slow oscillatory activity $(<1 \mathrm{~Hz})$ and wave propagations in a cortical network model,” J. Neurophysiol. vol. 89, pp. 2707-2725, 2003.

[77] D. T. Liley, D. M. Alexander, J. J. Wright, M. D. Aldous, “Alpha rhythm emerges from large-scale networks of realistically coupled multicompartmental model cortical neurons," Network, vol. 10, pp. 7992, 1999.

[78] S. R. Jones, D. J. Pinto, T. J. Kaper and N. Kopell, "Alpha-frequency rhythms desynchronise over long cortical distances: a modelling study," J. Comput. Neurosci., vol. 9, pp. 271-291, 2000

[79] Z. Li, "Contextual influences in V1 as a basis for pop out and asymmetry in visual search,” Proc. Nat. Acad. Sci., vol. 96, pp. 10530-10535, 1999.

[80] R. D. Raizada, S. Grossberg, "Towards a theory of the laminar architecture of cerebral cortex: computational clues from the visual system,” Cereb. Cortex., vol. 13(1), pp. 100-113, 2003.

[81] M. Wilson, J. M. Bower, "Cortical oscillations and temporal interactions in a computer simulation of piriform cortex," J. Neurophysiol., vol. 67(4), pp. 981-995, 1992.

[82] H. Liljenstrom, M. E. Hasselmo, "Cholinergic modulation of cortical oscillatory dynamics,” J. Neurophysiol., vol. 74, pp. 288-297, 1995.

[83] S. Basu, H. Liljenstrom, "Spontaneously active cells induce state transitions in a model of olfactory cortex," Biosystems, vol. 63, pp. 5769, 2001.

[84] L. Itti, C. Koch, "Computational modelling of visual attention," Nat. Rev. Neurosci., vol. 2, pp. 194-203, 2001.

[85] S. Corchs, G. Deco, "Large-scale neural model for visual attention: integration of experimental single-cell and fMRI data," Cereb. Cortex, vol. 12, pp. 339-348, 2002.

[86] A. Compte, N. Brunel, P. S., Goldman-Rakic, X. J. Wang, "Synaptic mechanisms and network dynamics underlying spatial working memory in a cortical network model,“ Cereb. Cortex, vol. 10, pp. 910-923, 2000.

[87] D. Durstewitz, J. K. Seamans, T. J. Sejnowski, "Neurocomputational models of working memory,” Nat. Neurosci., vol. 3, Suppl, 1184-1191, 2000.

[88] S. Kalitzin, B. W. van Dijk, H. Spekreijse, "Self-organized dynamics in plastic neural networks: bistability and coherence,” Biol. Cybern., vol. 83, pp. 139-150, 2000.

[89] N. Brunel, "Dynamics and plasticity of stimulus-selective persistent activity in cortical network models," Cereb. Cortex, vol. 13, pp. 11511161, 2003.

[90] D. A. McCormick, Y. Shu, A. Hasenstaub, M. Sanchez-Vives, M. Badoual, T. Bal, "Persistent cortical activity: mechanisms of generation and effects on neuronal excitability," Cereb. Cortex., vol. 13, pp. 1219$1231,2003$.

[91] G. Mongillo, E. Curti, S. Romani, D. J. Amit, "Learning in realistic networks of spiking neurons and spike-driven plastic synapses,” Eur. J. Neurosci., vol. 21, pp. 3143-3160, 2005

[92] R. A. Koene, M. E. Hasselmo, "An integrate-and-fire model of prefrontal cortex neuronal activity during performance of goal-directed decision making," Cereb. Cortex, to be published.

[93] G. Deco, E.T. Rolls, "Synaptic and spiking dynamics underlying reward reversal in the orbitofrontal cortex," Cereb. Cortex., vol. 15, 15-30, 2005.

[94] A. Destexhe, D. A. McCormick, T. J. Sejnowski, "A model for 8-10 Hz spindling in interconnected thalamic relay and reticularis neurons," Biophys J., vol. 65(6), pp. 2473-2477, 1993.

[95] X. J. Wang, J. Rinzel, "Spindle rhythmicity in the reticularis thalami nucleus: synchronization among mutually inhibitory neurons," Neuroscience, vol. 53(4), pp. 899-904, 1993.

[96] D. Golomb, X. J. Wang and J. Rinzel, "Synchronization properties of spindle oscillations in a thalamic reticular nucleus model" $J$. Neurophysiol., vol. 72, pp. 1109-1126, 1994.

[97] A. Destexhe, D. Contreras, T. J. Sejnowski and M. Steriade, "A model of spindle rhythmicity in the isolated thalamic reticular nucleus," $J$. Neurophysiol., vol. 72, pp. 803-818, 1994.

[98] M. Bazhenov, I. Timofeev, M. Steriade, and T. J. Sejnowski, "Selfsustained rhythmic activity in the thalamic reticular nucleus mediated by depolarizing GABAA receptor potentials," Nature Neurosci., vol. 2, pp. 168-174, 1999.

[99] X. J. Wang, D. Golomb, J. Rinzel, "Emergent spindle oscillations and intermittent burst firing in a thalamic model: specific neuronal mechanisms,” Proc. Nat. Acad. Sci. vol. 92(12), pp. 5577-5581, 1995.

[100]D. Golomb, X. J. Wang and J. Rinzel, "Propagation of spindle waves in a thalamic slice model,” J. Neurophysiol., vol. 75, pp. 750-769, 1996.

[101]A. Destexhe, T. Bal, D. A. McCormick and T. J. Sejnowski, "Ionic mechanisms underlying synchronized oscillations and propagating waves in a model of ferret thalamic slices," J. Neurophysiol., vol. 76, pp. 2049-2070, 1996.

[102]W. W. Lytton, D. Contreras, A. Destexhe, M. Steriade, "Dynamic interactions determine partial thalamic quiescence in a computer network model of spike-and-wave seizures," J. Neurophysiol., vol. 77, pp. 16791696, 1997.

[103]E. Thomas and T. Grisar, "Increased synchrony with increase of a lowthreshold calcium conductance in a model thalamic network: a phaseshift mechanism,” Neural Comput., vol. 12, pp. 1553-1571, 2000.

[104]A. Destexhe, D. Contreras and M. Steriade, "Mechanisms underlying the synchronizing action of corticothalamic feedback through inhibition of thalamic relay cells,” J. Neurophysiol., vol. 79, pp. 999-1016, 1998.

[105]D. Terman, A. Bose and N. Koppell, "Functional reorganization in thalamocortical networks: transition between spindling and delta sleep rhythms," Proc. Nat. Acad. Sci., vol. 93, pp. 15417-15422, 1996.

[106]A. Destexhe, "Modeling corticothalamic feedback and the gating of the thalamus by the cerebral cortex," J. Physiol. (Paris), vol. 94, pp. 391410, 2000

[107]A. Destexhe, "Spike-and-wave oscillations based on the properties of GABA $_{B}$ receptors,” J. Neurosci., vol. 18, pp. 9099-9111, 1998.

[108]A. Destexhe, "Can $\mathrm{GABA}_{\mathrm{A}}$ conductances explain the fast oscillation frequency of absence seizures in rodents?,” Eur. J. Neurosci., vol. 11, pp. 2175-2181, 1999.

[109]I. Timofeev, M. Bazhenov, T. Sejnowski, M. Steriade, "Cortical hyperpolarization-activated depolarizing current takes part in the generation of focal paroxysmal activities,” Proc. Nat. Acad. Sci. vol. 99(14), pp. 9533-9537, 2002.

[110]R. D. Traub, D. Contreras, M. O. Cunningham, H. Murray, F. E. LeBeau, A. Roopun, A. Bibbig, W. B. Wilent, M. J. Higley and M. A. Whittington, "Single-column thalamocortical network model exhibiting gamma oscillations, sleep spindles, and epileptogenic bursts. $J$. Neurophysiol., vol. 93, pp. 2194-2232, 2005.

[111]S. Hill, G.Tononi, "Modeling sleep and wakefulness in the thalamocortical system,” J. Neurophysiol., vol. 93, pp. 1671-1698, 2005.

[112]M. Abeles Corticonics, Cambridge, England: Cambridge University Press, 1991.

[113]Rieke, D. Warland, R. de Ruyter van Steveninck and W. Bialek, Spikes: Exploring the Neural Code. Cambridge, MA: MIT Press, 1999. 
[114]W. Maass and C. M. Bishop, Pulsed Neural Networks, Cambridge: MIT Press, 2001.

[115]H. R. Wilson and J. D. Cowan, "Excitatory and inhibitory interactions in localized populations of model neurons,” Biophys. J., vol. 12, pp.1-24, 1972.

[116]W. J. Freeman, "Patterns of variation in waveform of averaged evoked potentials from prepyriform cortex of cats,” J. Neurophysiol., vol. 31, pp. 1-13, 1968.

[117]W. J. Freeman, "The electrical activity of a primary sensory cortex: analysis of EEG waves," Int. Rev. Neurobiol., vol. 11, pp. 53-119, 1963.

[118]W. J. Freeman, "A model of the olfactory system," in Neural modeling, M. A. B. Brazier, D. O. Walter, and D. Schneider, Eds. Los Angeles: Univ. of California, 1973, pp. 41-62.

[119]F. H. Eeckman, W. J. Freeman, "Asymmetric sigmoid non-linearity in the rat olfactory system,” Brain Res., vol. 23, pp. 557, pp. 13-21, 1991.

[120]W. J. Freeman, "Simulation of chaotic EEG patterns with a dynamic model of the olfactory system," Biol. Cybern., vol. 56, pp. 139-150, 1987.

[121]F.H. Lopes da Silva, A. Hoeks, H. Smits and L. H. Zetterberg, "Model of brain rhythmic activity. The alpha-rhythm of the thalamus," Kybernetic, vol. 15, pp. 27-37, 1974.

[122]A. van Rotterdam, F. H. Lopes da Silva, J. van den Ende, M. A. Viergever, A. J. Hermans, "A model of the spatial temporal characteristics of the alpha rhythm," Bulletin of Mathematical Biology, vol. 44 (2), pp. 238-305, 1982.

[123]L. H. Zetterberg, L. Kristiansson, K. Mossberg, "Performance of the model for a local neuron population,” Biol. Cybern., vol. 31, pp. 15-26, 1978.

[124]B. H. Jansen, V. G. Rit, "Electroencephalogram and visual evoked potential generation in a mathematical model of coupled cortical columns,” Biol. Cybern., vol. 73, pp. 357-366, 1995.

[125]F. Wendling, J. J. Bellanger, F. Bartolomei, P. Chauvel, "Relevance of nonlinear lumped-parameter models in the analysis of depth-EEG epileptic signals,” Biol. Cybern., vol. 83, pp. 367-378, 2000.

[126]F. Wendling, F. Bartolomei, J. J. Bellanger, P. Chauvel, „Epileptic fast activity can be explained by a model of impaired GABAergic dendritic inhibition,” Eur. J. Neurosci., vol. 15(9), pp. 1499-1508, 2002.

[127]P. Suffczynski, S. Kalitzin, G. Pfurtscheller and F. H. Lopes da Silva, "Computational model of thalamo-cortical networks: dynamical control of alpha rhythms in relation to focal attention," Int. J. Psychophysiol., vol. 43, pp. 25 - 40, 2001.

[128]P. Suffczynski, S. Kalitzin, F. H. Lopes da Silva, "Dynamics of nonconvulsive epileptic phenomena modeled by a bistable neuronal network,” Neuroscience, vol. 126(2), pp. 467-484, 2004.

[129]O. David, K. J. Friston, "A neural mass model for MEG/EEG: coupling and neuronal dynamics," Neuroimage, vol. 20, pp. 1743-1755, 2003.

[130]P. L. Nunez, Electric Fields of the Brain: The Neurophysics of EEG. New York: Oxford University Press, 1981.

[131]P. L. Nunez, "A study of origins of the time dependencies of scalp EEG: i-theoretical basis," IEEE Trans. Biomed. Eng., vol. 28(3), pp. 271-280, 1981.

[132]P. L. Nunez, "Generation of human EEG by a combination of long and short range neocortical interactions," Brain Topogr., vol. 1(3), pp. 199215, 1989.

[133]P. L. Nunez, Neocortical Dynamics and Human EEG Rhythms. New York: Oxford University Press, 1995.

[134]P. L. Nunez, "A study of origins of the time dependencies of scalp EEG: ii--experimental support of theory," IEEE Trans. Biomed. Eng., vol. 28(3), pp. 281-288, 1981.

[135]G. R. Burkitt, R. B.Silberstein , P. J. Cadusch, A. W. Wood, "Steadystate visual evoked potentials and travelling waves," Clin. Neurophysiol., vol. 111, pp. 246-58, 2000.

[136]J. J. Wright and D. T. J. Liley, "Simulation of electrocortical waves," Biol. Cybern., vol. 72, pp. 347-356, 1995.

[137]J. J. Wright, P. D. Bourke, C. L. Chapman, "Synchronous oscillation in the cerebral cortex and object coherence: simulation of basic electrophysiological findings,” Biol. Cybern., vol. 83, pp. 341 - 353, 2000.

[138]P. A. Robinson, C. J. Rennie, J. J. Wright, "Propagation and stability of waves of electrical activity in the cerebral cortex," Phys. Rev. E., vol. 56, pp. 826-840, 1997.
[139]P. A. Robinson P. N. Loxley, S. C. O'Connor, C.J. Rennie, "Modal analysis of corticothalamic dynamics, electroencephalographic spectra, and evoked potentials,” Phys. Rev. E., vol. 63, pp. 1 - 13, 2001.

[140]C. J. Rennie, P. A. Robinson and J. J. Wright, "Unified neurophysical model of EEG spectra and evoked potentials," Biol. Cybern., vol. 86, 457-471, 2002.

[141]P. A. Robinson, C. J. Rennie, and D. L. Rowe, "Dynamics of large-scale brain activity in normal arousal states and epileptic seizures," Phys. Rev. E., vol. 65, pp. 1-9, 2002.

[142]J. J. Wright, D. T. J. Liley, "Dynamics of the brain at global and microscopic scales. Neural networks and the EEG," Behavioral and Brain Sciences, vol. 19, pp. 285-320, 1996.

[143]F.H. Lopes da Silva, W. Blanes, S. N. Kalitzin, J. Parra, P. Suffczynski, D. N. Velis, "Dynamical diseases of brain systems: different routes to epileptic seizures," IEEE Trans. Biomed. Eng., vol. 50, pp. 540 - 548, 2003.

[144]J. French, P. Williamson, V. Thadani, T. Darcey, R. Mattson, S. Spencer, D. Spencer, "Characteristics of medial temporal lobe epilepsy: I. Results of history and physical examination,” Ann. Neurol., vol. 34, pp. 774-780, 1993.

[145]P. Williamson, J. French, V. Thadani, J. Kim, R. Novelly, S. Spencer, D. Spencer, R. Mattson, "Characteristics of medial temporal lobe epilepsy: II. Interictal and ictal scalp electroencephalography, neuropsychological testing, neuroimaging, surgical results and pathology,” Ann. Neurol., vol. 34, pp. 781-787, 1993.

[146]J. Engel, P. van Ness, T. Rasmussen, L. Ojemann, "Outcome with respect to epileptic seizures," in Surgical treatment of the epilepsies, 2nd ed., J. Engel, Ed. New York, NY: Raven Press, 1993, pp. 609-622.

[147]M. A. Whittington, R. D. Traub, N. Kopell, B. Ermentrout and E. H. Buhl, "Inhibition-based rhythms: experimental and mathematical observations on network dynamics," Int. J. Psychophysiol., vol. 38, 315336, 2000.

[148]R. Cossart, C. Dinocourt, J. C. Hirsch, A. Merchan-Perez, J. De Felipe, Y. Ben-Ari, M. Escalpez, C. Bernard, "Dendritic but not somatic GABAergic inhibition is decreased in experimental epilepsy," Nat. Neurosci., vol. 4(1), pp. 52-62, 2001.

[149]M. I. Banks, J. A. White and R. A. Pearce, "Interactions between distinct GABA(A) circuits in hippocampus,” Neuron, vol. 25, pp. 449-457, 2000

[150]A. Destexhe, D. Contreras, M. Steriade, "LTS in cerebral cortex and their role in generating spike-and-wave oscillations," Neurocomputing, vol. 38-40, pp. 555-563, 2001.

[151]P. Suffczynski, F. H. Lopes da Silva, J. Parra, D. Velis, B. M. Bouwman, C. M. van Rijn, P. van Hese, P. Boon, H. Khosravani, M. Derchansky, P. Carlen, and S. Kalitzin, "Dynamics of epileptic phenomena determined from statistics of ictal transitions," IEEE Trans. Biomed. Eng., to be published.

[152]H. K. Meeren, J. P. Pijn, E. L. Van Luijtelaar, A. M. Coenen, F. H. Lopes da Silva, "Cortical focus drives widespread corticothalamic networks during spontaneous absence seizures in rats,” J. Neurosci., vol. 22(4), pp. 1480-1495, 2002.

[153]M. L. Hines and N. T. Carnevale, "The NEURON simulation environment" in The Handbook of Brain Theory and Neural Networks, 2nd ed. M. A. Arbib, Ed., Cambridge, MA, MIT Press, 2003.

[154]J. M. Bower, D. Beeman, The book of GENESIS: exploring realistic neural models with the GEneral NEural SImulation System. New York: Springer-Verlag, 1998.

[155]M. Hines, "Efficient computation of branched nerve equations," Int. J. Bio-Med. Comput., vol. 15, pp. 69-76, 1984.

[156]E. De Schutter, "A consumer guide to neuronal modeling software," Trends Neurosci., vol. 15, pp. 462-464, 1992.

[157]A. Weitzenfeld, M. Arbib, A. Alexander, The Neural Simulation Language: A System for Brain Modeling, Cambridge, MA: MIT Press, 2002

[158]C. M. Lloyd, M. D. B. Halstead and P. F. Nielsen, "CellML: its future, present and past," Progress in Biophysics and Molecular Biology, vol. 85, pp. 433-450, 2004.

[159]A. A. Prinz, L. F. Abbott, E. Marder, "The dynamic clamp comes of age,” Trends Neurosci., vol. 27(4), pp. 218-224, 2004.

[160]M. Hutzler, P. Fromherz, "Silicon chip with capacitors and transistors for interfacing organotypic brain slice of rat hippocampus," Eur. J. Neurosci., vol. 19, pp. 2231-2238, 2004. 
[161]P. Fromherz, “The neuron-semiconductor interface," in Bioelectronics from Theory to Applications, I. Willner and E. Katz, Ed. Weinheim: Wiley-VCH, 2005, pp. 339-394.

[162]R. Douglas, M. Mahowald, C. Mead, "Neuromorphic analogue VLSI," Annu. Rev. Neurosci., vol. 18, pp. 255-281, 1995.

[163]A. van Schaik, R. Meddis, “Analog very large-scale integrated (VLSI) implementation of a model of amplitude-modulation sensitivity in the auditory brainstem,“ J. Acoust. Soc. Am., vol. 105, pp. 811-821, 1999.

[164]S. C. Liu, J. Kramer, G. Indiveri, T. Delbruck, T. Burg, R. Douglas, “Orientation-selective aVLSI spiking neurons," Neural Netw., vol. 14, pp. 629-643, 2001.

[165]T. Delbruck, S. C. Liu, "A silicon early visual system as a model animal,” Vision Res., vol. 44, pp. 2083-2089, 2004.

[166]K. Eng, D. Klein, A. Babler, U. Bernardet, M. Blanchard, M. Costa, T. Delbruck, R. J. Douglas, K. Hepp, J. Manzolli, M. Mintz, F. Roth, U. Rutishauser, K. Wassermann, A. M. Whatley, A. Wittmann, R. Wyss, P. F. Verschure, "Design for a brain revisited: the neuromorphic design and functionality of the interactive space 'Ada'," Rev. Neurosci., vol. 14, pp. 145-180, 2003.

[167]A. A. Prinz, D. Bucher, E. Marder, "Similar network activity from disparate circuit parameters,” Nat. Neurosci., vol. 7(12), 1345-1352, 2004.

[168]J. M. Bower, C. Koch, "Experimentalists and modelers: can we all just get along?," Trends. Neurosci., vol. 15, 458-61, 1992.

[169]M. C. Casdagli, L. D. Iasemidis, R. S. Savit, R. L. Gilmore, S. N. Roper, J. C. Sackellares, "Non-linearity in invasive EEG recordings from patients with temporal lobe epilepsy. Electroenceph. Clin. Neurophysiol., vol. 102, pp. 98-105, 1997.

[170]K. Linkenkaer-Hansen, V. V. Nikouline, J. M. Palva, R. J. Ilmoniemi, "Long-range temporal correlations and scaling behavior in human brain oscillations,“ J. Neurosci., vol. 21, pp. 1370-1377, 2001.

[171]H. Khosravani, P. L. Carlen, J. L. Velazquez, "The control of seizurelike activity in the rat hippocampal slice," Biophys. J., vol. 84, pp. 687695, 2003.

[172]L. M. Parish, G. A. Worrell, S. D. Cranstoun, S. M. Stead, P. Pennell, B. Litt, "Long-range temporal correlations in epileptogenic and nonepileptogenic human hippocampus,” Neuroscience, pp. 125(4), pp. 1069-1076, 2004. 
Legends

Figure 1: Different levels of neuronal organization. a) The brain consists of various systems (e.g. visual, auditory, somatosensory) that mediate a specific behavior. The scheme shows lateral view of the brain with anatomical divisions into brain lobes. b) Each system consists of local populations, i.e., densely interconnected networks of neurons. c) A typical nerve cell (neuron) consists of dendritic tree, cell body (soma) and axon. Neuron receives a number of synaptic contacts, mainly on the dendritic tree or the cell soma. Arriving action potential (arrows) initiates generation of either excitatory or inhibitory synaptic potential that spreads passively to the cell body. If the summed amplitude of synaptic potentials at the initial segment of the axon exceeds the threshold, action potential is generated that propagates down the axon and causes a transmitter release that triggers a postsynaptic potential in the target (postsynaptic) cell. d) Synaptic transmission involves many complex processes like release of neurotransmitter and activation of receptors in the postsynaptic cell that enables the flow of ions through channels in the postsynaptic membrane. e) Neuron's membrane contains voltage- and ligand-gated channels through which charged ions can flow altering membrane potential what allows a neuron to signal and respond to other cells. f) Genes contain the instructions for the production of proteins, which make up the structure of cells and direct their activities. (Fig. modified from [1]).

Figure 2: Equivalent electrical circuit corresponding to a piece of membrane of length $d x$. Quantities $r_{i}, r_{m}, C_{m}, r_{K}, r_{N a}$ are defined for a unit length. Resistance $r_{m}$ is the total passive (non voltage-dependent) resistance across the membrane per unit length. Associated characteristics are $R_{m}=\pi d r_{m}$ (where $\mathrm{d}$ is cable diameter) equal to the resistance presented by a membrane unit area and $R_{i}=\pi d^{2} r_{i} / 4$ equal to the intracellular resistivity. $I_{i}$ is the intracellular longitudinal current. $I_{c}$ corresponds to the charge current in membrane capacity. In Rall's model (a), the membrane behaves as a passive cable. The Hodgkin and Huxley's model (b) adds the dependence of the membrane potential on sodium and potassium currents through corresponding voltage-dependent ionic channels. $G_{K}=$ $1 / r_{K}$ and $G_{N a}=1 / r_{N a}$ are the voltage- and time-dependent membrane conductances.

Figure 3: Neuronal population model. a) The model canonical form reproduces interactions between main cells (pyramidal) and local excitatory neurons or inhibitory interneurons. In each subpopulation, a static nonlinear function (asymmetric sigmoid curve $\left.S(v)=2 e_{0} /\left[1+e^{r\left(v_{0}-v\right)}\right]\right)$ is used to model threshold and saturation effects in the relationship between the average post-synaptic potential of a given subset and the average pulse density of potentials fired by the neurons. In turn, a linear transfer function is used to transform the average pre-synaptic pulse density of afferent action potentials (the input) into an average post-synaptic membrane potential (the output). This transfer function is of order 2, as described in section V.B. Interneurons receive an excitatory input (AMPA and NMDA receptor-mediated) from pyramidal cells. The associated impulse response $h_{E X C}(t)=E X C$.a.t.e $e^{-a t}$ determines the excitatory average post-synaptic membrane potential, where EXC represent the synaptic gain. Similarly, interneurons

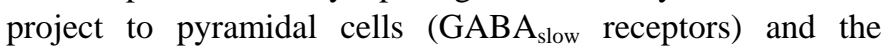
impulse response $h_{S D I}(t)=S D I . b . t . e^{-b t}$ determines the inhibitory average post-synaptic membrane potential (lower feedback loop). Recurrent excitation (excitatory-excitatory connections between pyramidal cells) is also represented in the model (upper feedback loop). The influence from neighboring or more distant populations is represented by an excitatory input $n(t)$ (modeled by a positive mean gaussian white noise) that globally describes the average density of afferent action potentials. The average number of synaptic contacts between subsets of cells is represented by connectivity constants C1 to C4. Model output corresponds to the post-synaptic activity of the subset of pyramidal cells that mainly contributes to the EEG signal (field potential). b) Modified model designed to represent the cellular organization in the hippocampus (CA1). A subset of somaticprojecting interneurons (grey rectangle) was added to the previous model. Consistently with ([148]), this subpopulation receives input from both subsets of pyramidal and dendriticprojecting interneurons. Similarly to other subpopulations, a linear transfer function is used to convert the pre-synaptic pulse density into a post-synaptic membrane potential. Its impulse response, $h_{F S I}(t)=F S I . g . t . e^{-g t}$, determines fast somatic inhibitory average post-synaptic membrane potentials. c) The different types of activity produced by the model and comparison with b) real depth-EEG signals recorded in human hippocampus (during stereoelectroencephalographic exploration and using intracerebral multiple lead depthelectrodes). Type 1 and type 2, respectively, refer to normal background activity and sporadic spikes as observed in real signals during interictal periods. Type 3 and type 4, respectively, refer to sustained spiking activity and slow rhythmic activity. Both may be observed in the hippocampus just before seizure onset. Type 5 refers to fast activity that appears at seizure onset. Finally, type 6 refers to slower narrow-band activity.

Figure 4: Model-based interpretation of EEG signals during the transition to seizure in TLE. (a) intracerebral recording in human hippocampus. Five phases (1 to 5) are distinguished according to the pseudo-stationary nature of the activities reflected by the signal: interictal activity (1), high-amplitude spikes during preonset period $(2,3)$, rapid discharge (4) at seizure onset and quasi-sinusoidal ictal activity (5). b) (SDI, FSI) plane for given constant parameter EXC obtained from model parameter sensitivity study and a candidate path in the parameter space that explains the transitions of activity observed in the real signal. c) Evolution of slow and fast 
inhibition parameters (SDI, FSI) defined from the candidate path and used in the model to simulate a time-series signal (d). Transitions in dynamics match those observed. e) Normalized power spectral densities of real (solid line) and simulated (dotted line) signals.

Figure 5: Thalamocortical population model. a) Schematic diagram of connections between thalamocortical relay (TC) cells, cells of reticular nucleus (RE), cortical pyramidal (PY) cells and cortical interneurons (IN). The thalamocortical cells receive input from ascending specific afferents and project to a localized region of the cortex and to the reticular nucleus neurons. The RE cells are connected to each other by means of dendrodendritic synapses and send back GABAergic inhibitory fibers to the TC cells. PY cells send feedback connection to both types of thalamic cells and to the interneurons. The relay nucleus receives cholinergic modulatory input from the brain stem while the reticular nucleus receives also cholinergic input, both from the brain stem as well as from the basal forebrain. Open triangles mark excitatory connections and closed circles, inhibitory ones. b) The general block diagram of the thalamocortical model based on the structure of thalamocortical network. c) Example of the model output. Upper panel: 20 seconds of a simulation with the occurrence of a spontaneous paroxysmal episode. Lower panels: power spectra of simulated normal and paroxysmal activity in the model and in the epileptic rat. Dominant frequency of normal activity is around $11 \mathrm{~Hz}$ while that of paroxysmal activity is around $9 \mathrm{~Hz}$. d) Durations distributions of the ictal (upper graph) and interictal (lower graph) epochs generated during 24 simulation of the model. Exponential fit is shown in both pictures indicating that both seizure termination (upper graph) and initiation (lower graph) occur randomly over time.

Figure 6: Number of publications per year in neuroscience and computational neuroscience fields, showing different dynamics (linear increase vs. exponential growth) of these two disciplines. Rough estimation of a number of publications was made using PubMed database (http://www.ncbi.nlm.nih.gov/). Papers that contained the word 'brain' (i.e, matched 'brain AND year[edat]' query) were considered as neuroscience articles published in the respective year. Papers that contained the words 'brain' and 'computer model' (i.e., matched 'brain AND computer model AND year[edat]' query) were considered as computational neuroscience articles published in the respective year. 
Figure 1

(a)

(b)

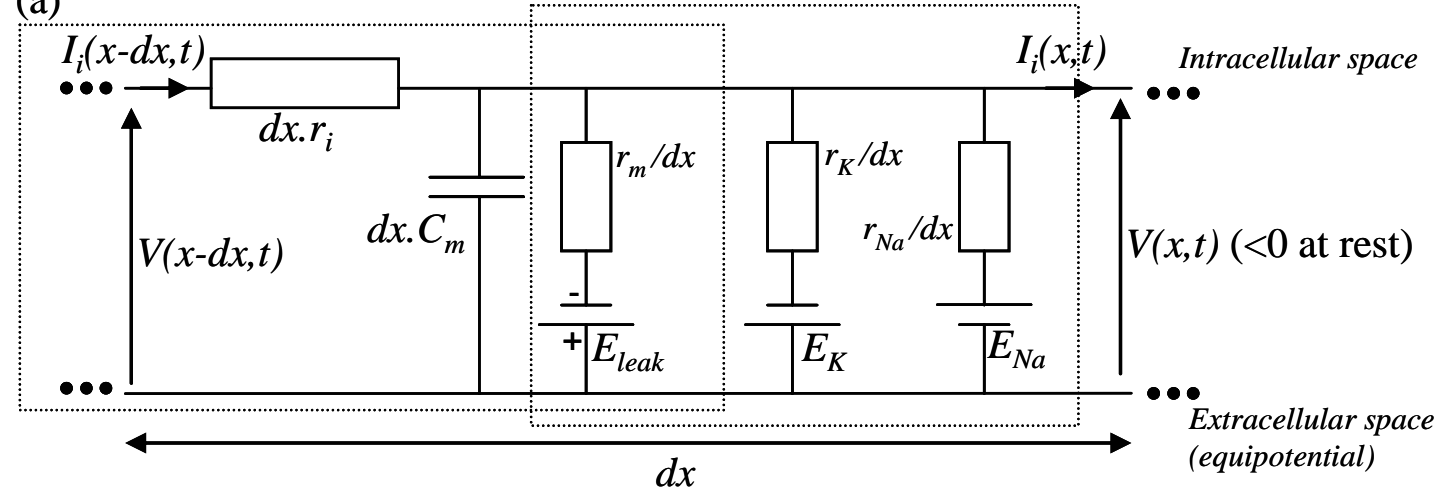


Figure 2

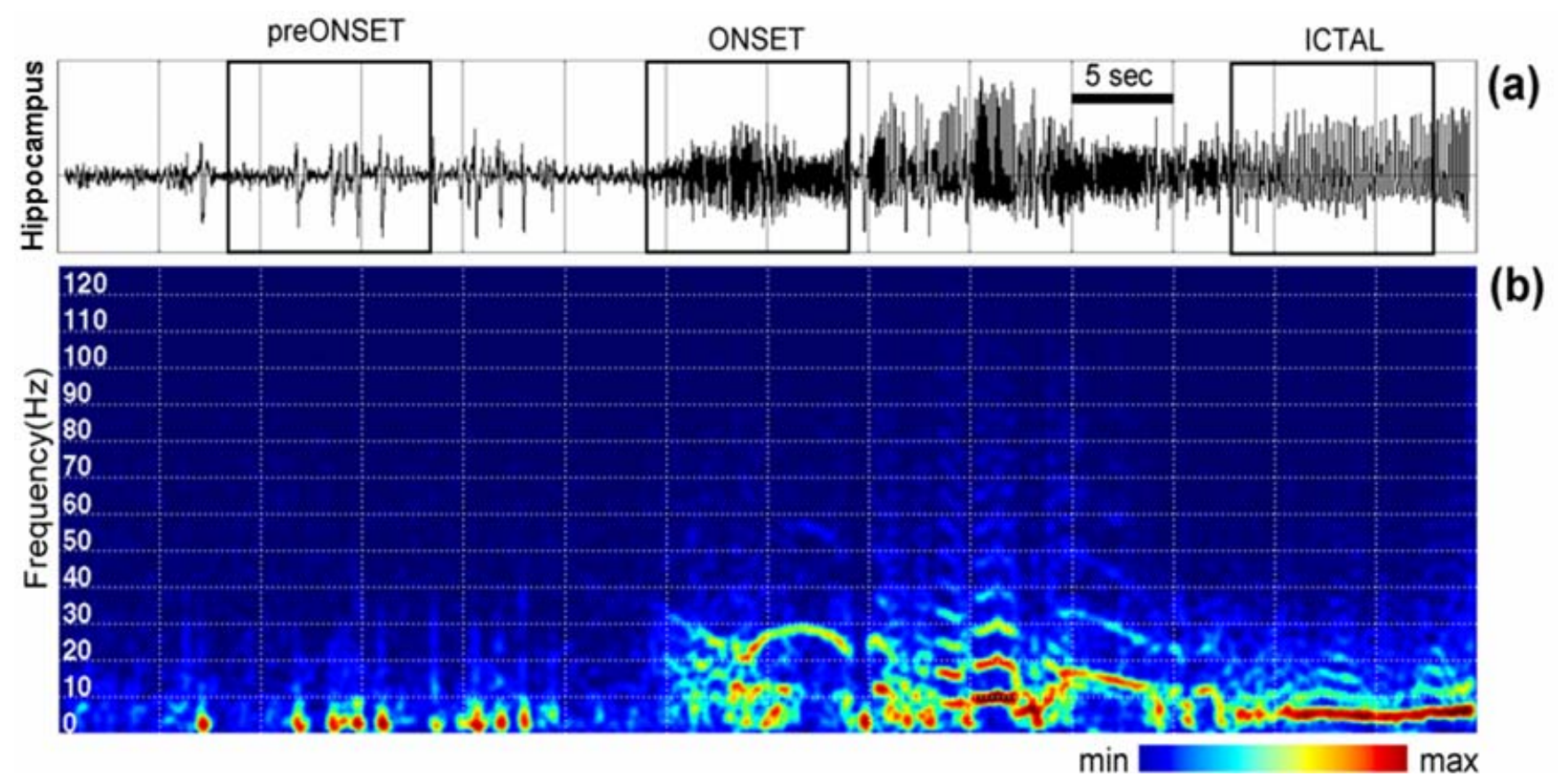


Figure 3
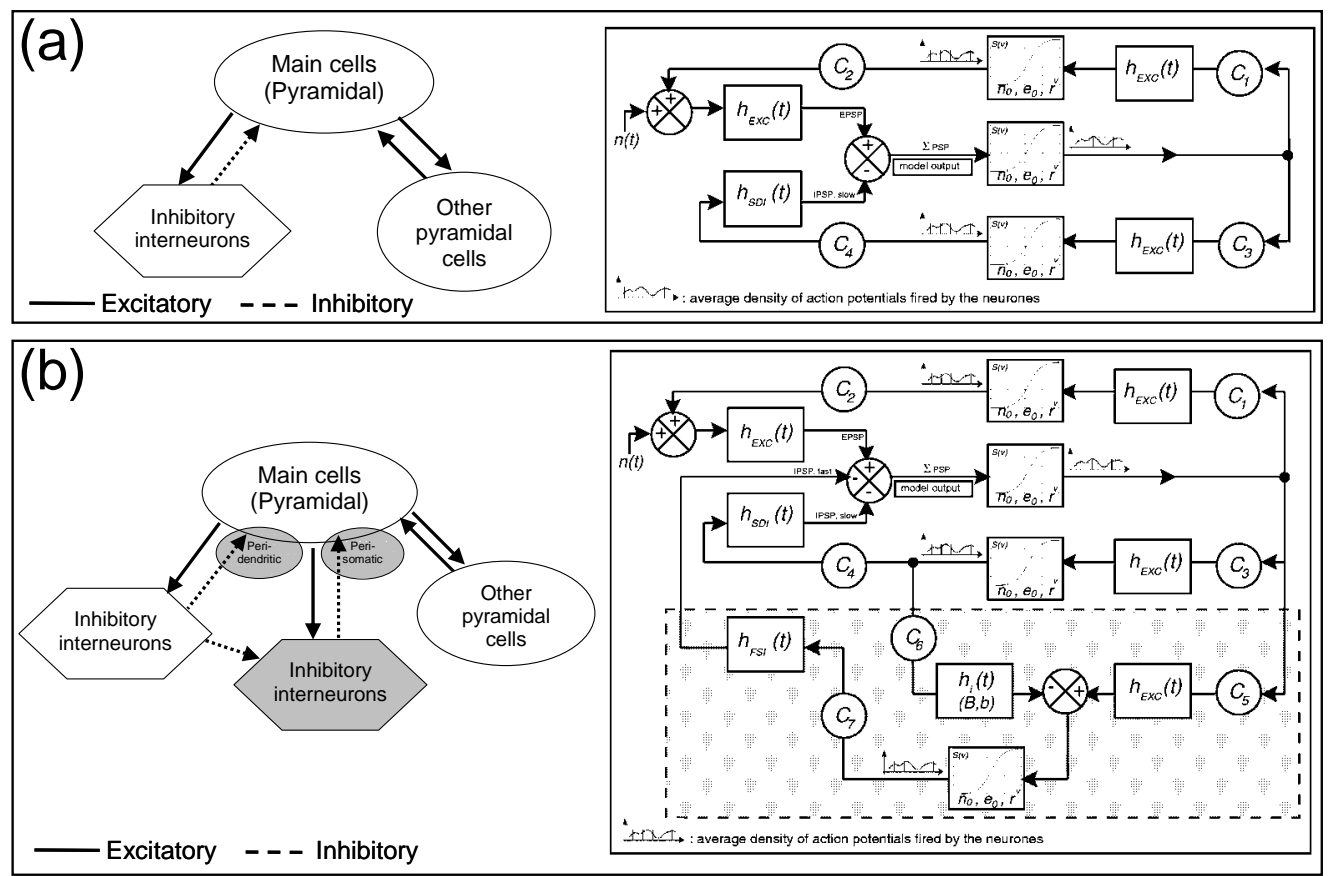

(C) Simulated signals
Type 1


Figure 4
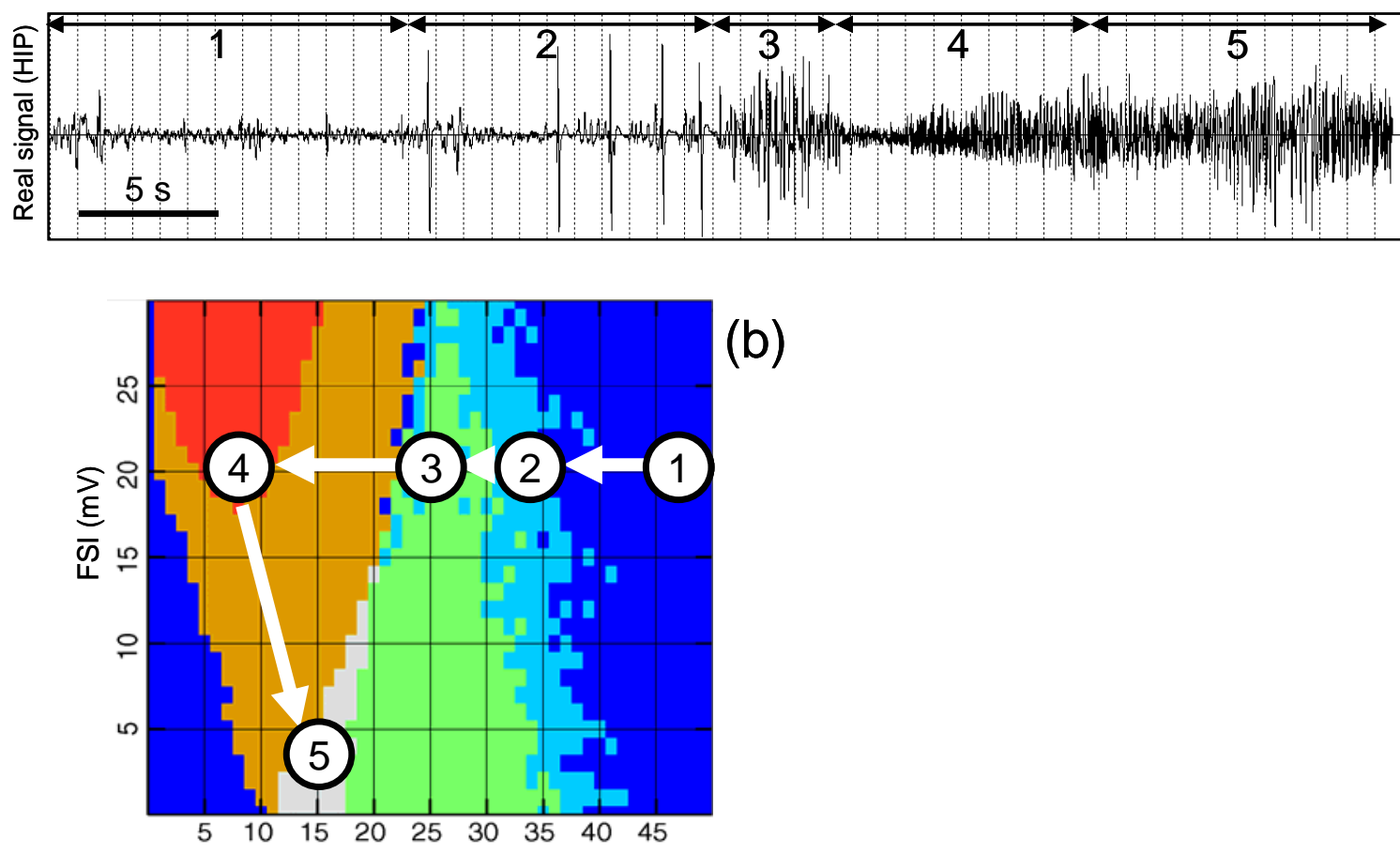

(b)
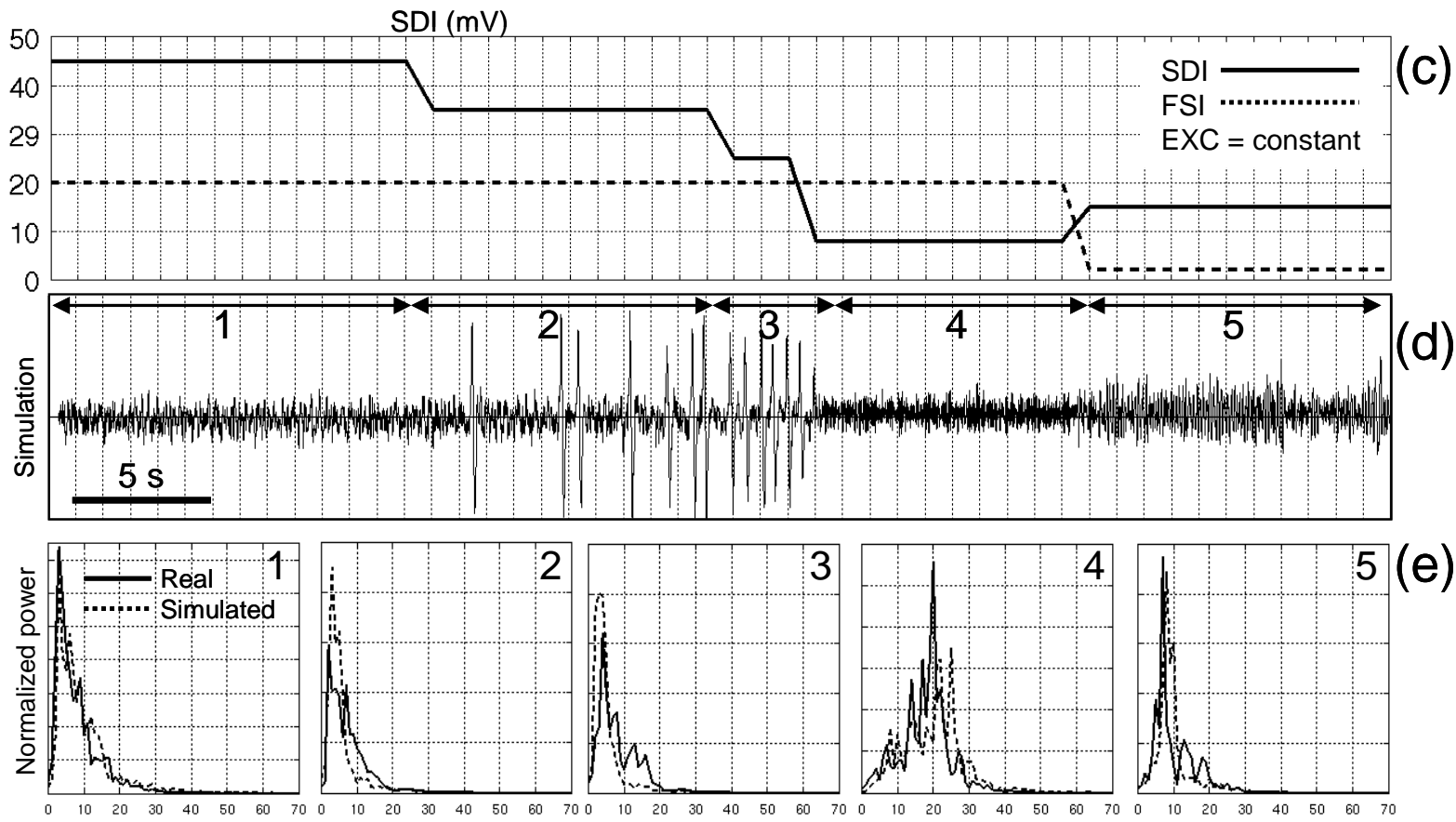

$5(e)$ Frequency $(\mathrm{Hz})$ 
Figure 5

\section{(a) anatomy}

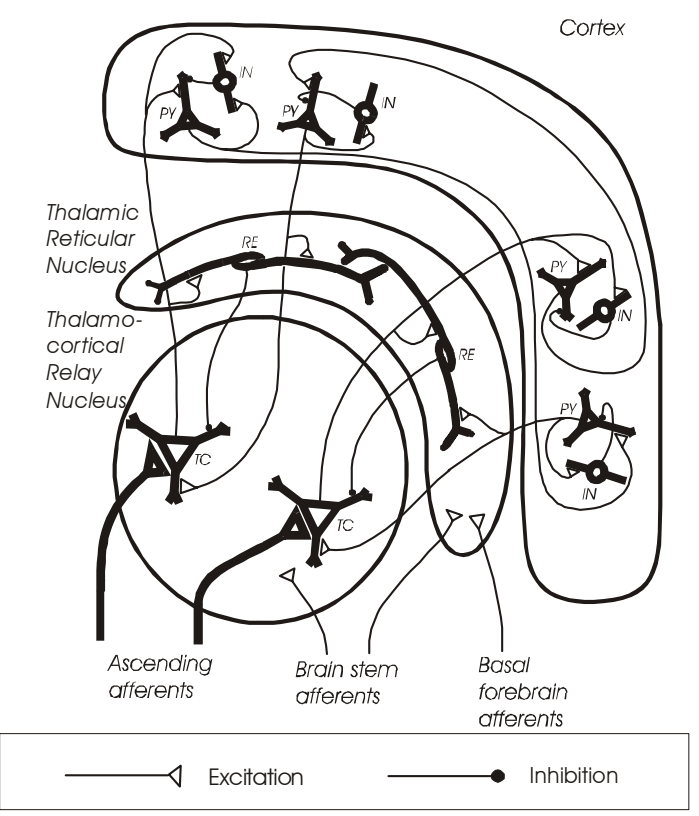

(c) simulated signals

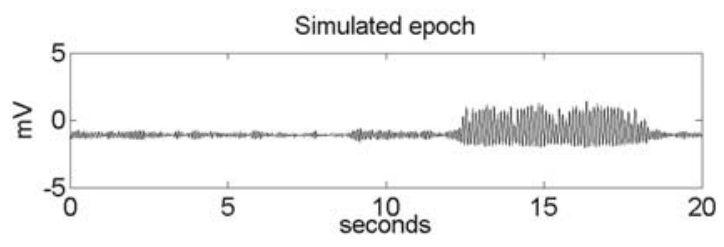

Power spectra
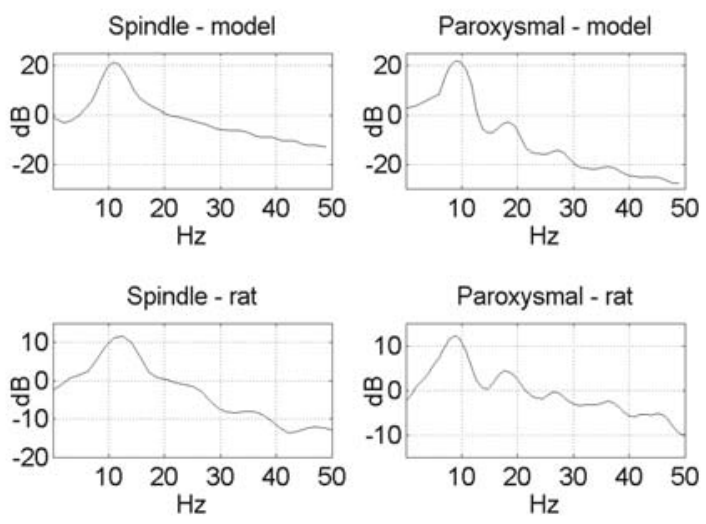

(b) model scheme

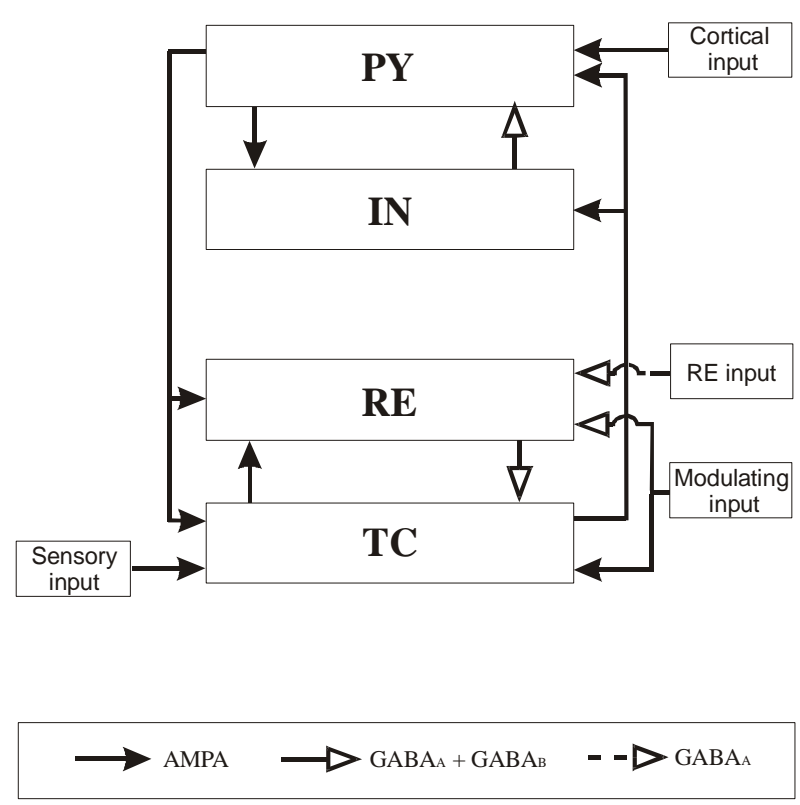

(d) duration distributions
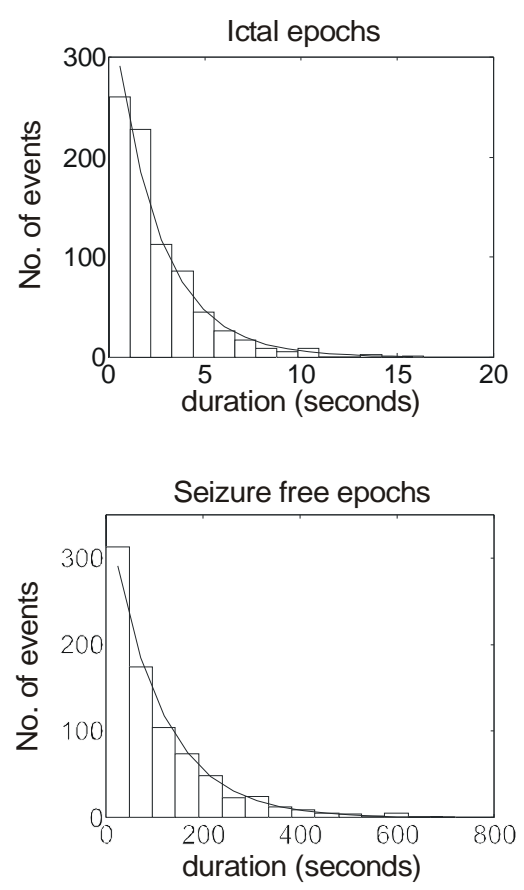
Figure 6

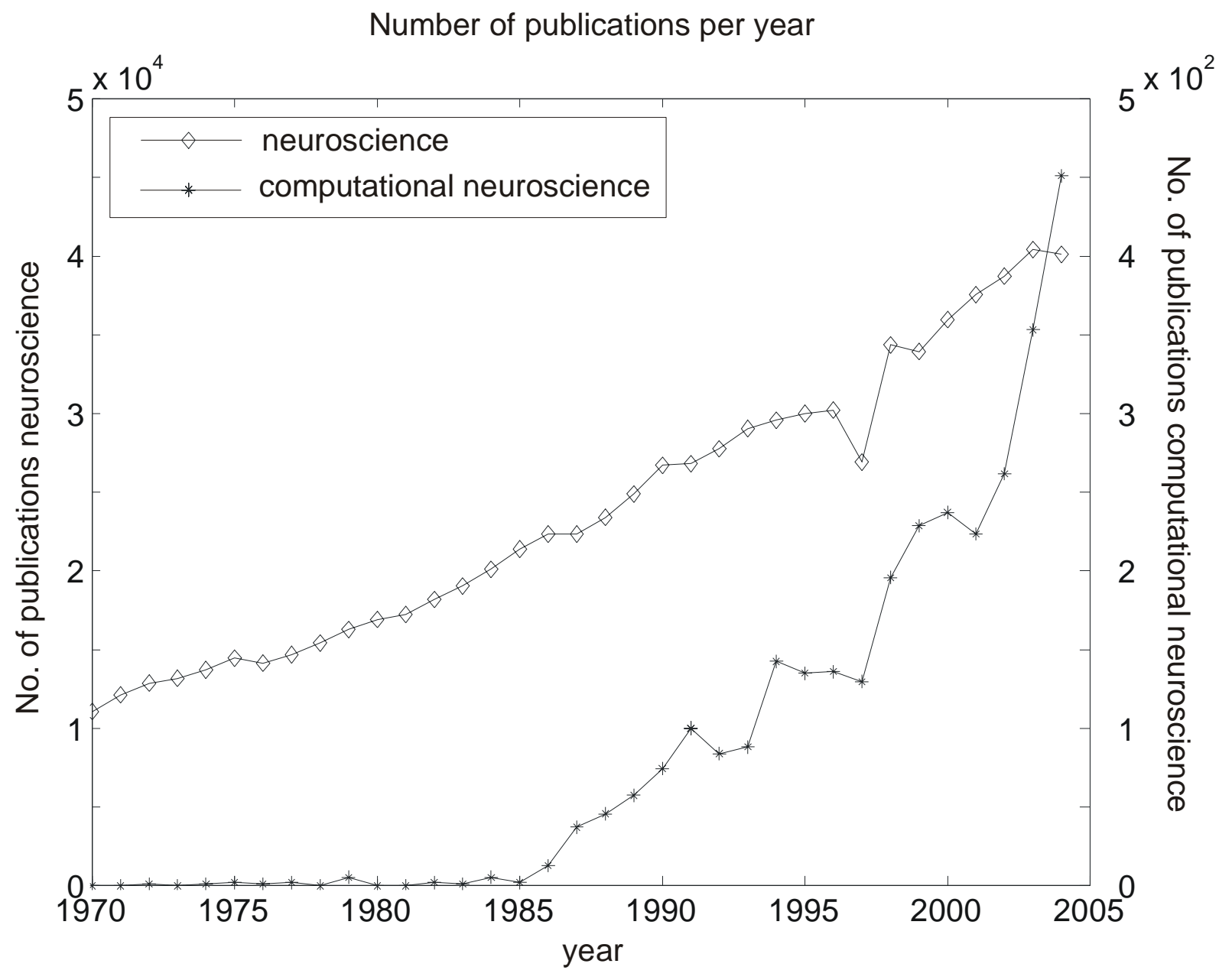

